ARTICLE

DOI: $10.1038 / s 41467-017-01218-8$

\title{
Structural basis of arrestin-3 activation and signaling
}

Qiuyan Chen ${ }^{1}$, Nicole A. Perry' ${ }^{1}$ Sergey A. Vishnivetskiy ${ }^{1}$, Sandra Berndt ${ }^{1}$, Nathaniel C. Gilbert ${ }^{1,9}$, Ya Zhuo $^{2}$, Prashant K. Singh ${ }^{3}$, Jonas Tholen4, Melanie D. Ohi 3,5,6, Eugenia V. Gurevich ${ }^{1}$, Chad A. Brautigam, Candice S. Klug ${ }^{2}$, Vsevolod V. Gurevich (iD ${ }^{1}$ \& T.M. Iverson (1) 1,5,6,8

A unique aspect of arrestin-3 is its ability to support both receptor-dependent and receptorindependent signaling. Here, we show that inositol hexakisphosphate $\left(\mathrm{IP}_{6}\right)$ is a non-receptor activator of arrestin- 3 and report the structure of $\mathrm{IP}_{6}$-activated arrestin-3 at $2.4-\AA$ resolution. $\mathrm{IP}_{6}$-activated arrestin-3 exhibits an inter-domain twist and a displaced C-tail, hallmarks of active arrestin. $I_{6}$ binds to the arrestin phosphate sensor, and is stabilized by trimerization. Analysis of the trimerization surface, which is also the receptor-binding surface, suggests a feature called the finger loop as a key region of the activation sensor. We show that finger loop helicity and flexibility may underlie coupling to hundreds of diverse receptors and also promote arrestin-3 activation by $\mathbb{I P}_{6}$. Importantly, we show that effector-binding sites on arrestins have distinct conformations in the basal and activated states, acting as switch regions. These switch regions may work with the inter-domain twist to initiate and direct arrestin-mediated signaling.

\footnotetext{
${ }^{1}$ Department of Pharmacology, Vanderbilt University, Nashville, TN 37232, USA. ${ }^{2}$ Department of Biophysics, Medical College of Wisconsin, Milwaukee, WI 53226, USA. ${ }^{3}$ Department of Cell and Developmental Biology, Vanderbilt University, Nashville, TN 37232, USA. ${ }^{4}$ University of Applied Sciences Emden/ Leer, Emden, 26723, Germany. ${ }^{5}$ Department of Biochemistry, Vanderbilt University, Nashville, TN 37232, USA. ${ }^{6}$ Center for Structural Biology, Vanderbilt University, Nashville, TN 37232, USA. 7 Departments of Biophysics and Microbiology, The University of Texas Southwestern Medical Center, Dallas, TX 75390, USA. ${ }^{8}$ Vanderbilt Institute of Chemical Biology, Vanderbilt University, Nashville, TN 37232, USA. ${ }^{9}$ Present address: Louisiana State University, Baton Rouge, LA 70803, USA. Correspondence and requests for materials should be addressed to V.V.G. (email: vsevolod.gurevich@vanderbilt.edu) or to T.M.I. (email: tina.iverson@vanderbilt.edu)
} 
A rrestins modulate $G$ protein-coupled receptor (GPCR) signaling in two ways. First, arrestins bind to activated, phosphorylated receptor and sterically block $G$ protein coupling, terminating $G$ protein activation ${ }^{1}$. Second, receptorbound non-visual arrestins (arrestin- 2 and -3, a.k.a. $\beta$-arrestin-1 and -2 ) initiate $G$ protein-independent signaling ${ }^{2}$ via $>100$ proteins ${ }^{3}$. This arrestin-mediated signaling regulates cell proliferation and apoptosis ${ }^{2}$.

GPCR-dependent arrestin signaling occurs in the context of a complex between arrestin and phosphorylated, activated GPCR. In classic biochemical work on rhodopsin and arrestin-1, this arrestin-receptor interaction was found to rely on arrestin "sensors" that detect the phosphorylation and activation of the receptors. The phosphate sensor is formed by a group of positively charged side chains on the concave side of the $\mathrm{N}$-domain that directly bind receptor-attached phosphates and is relatively unselective for the underlying sequence ${ }^{4}$. The activation sensor distinguishes between active and inactive GPCRs. To our knowledge, neither the identity of the activation sensor nor how it recognizes >800 distinct GPCRs have been proposed. These phosphate and activation sensors are believed to act synergistically, as the simultaneous triggering of both sensors elicits the highest-affinity receptor binding ${ }^{5}$.

Structural studies in the context of biochemical work suggest that triggering the phosphate and activation sensors of arrestin promotes a conformational change. Structures of all four vertebrate arrestins in the basal state $e^{4,6-8}$ showed that arrestin is a two-domain protein with limited interaction between domains. Two inter-domain interactions have been suggested as key for stabilizing the basal conformation. The first is a group of buried charged side chains known as the "polar core" 7 . The second is a sequence in the C-terminus of arrestin termed the "C-tail" (residues 385-393 of arrestin-3) that binds to the $\mathrm{N}$-domain in a way that both contributes a charge to the polar core and blocks access to the phosphate sensor ${ }^{7}$. Perturbation of either the polar core or the C-tail shifts the arrestin equilibrium to favor activation, suggesting that the inter-domain arrangement changes during activation ${ }^{9}$.

Recently, several strategies to stabilize active arrestin allowed structural characterization. These included the use of: (1) a more easily activated (termed "pre-activated") splice variant of visual arrestin- 1 called $\mathrm{p} 44^{10}$, which is truncated before the C-tail; (2) an antibody to stabilize arrestin-2 bound to a vasopressin receptor phosphopeptide ${ }^{11}$; (3) the R175E mutant of arrestin-1 which has a destabilized polar core ${ }^{12}$; and (4) arrestin-1 with activating mutations tethered to constitutively active rhodopsin ${ }^{13}$. In the structures of $\mathrm{p} 44$, the phosphopeptide-bound arrestin-2, and the receptor-bound arrestin- $1, \mathrm{a} \sim 20^{\circ}$ inter-domain rotation was observed, identifying inter-domain rotation as a hallmark of arrestin activation ${ }^{10,11,13}$. In the R175E variant, a $\sim 7.5^{\circ}$ interdomain rotation suggests that this structure represents an activation intermediate ${ }^{12}$.

Despite these advances, many fundamental questions in arrestin-mediated signaling remain unanswered. Non-visual arrestins bind $>800$ distinct GPCRs, yet it is not clear how this broad receptor specificity is achieved. It is also unclear how the phosphate and activation sensors elicit the inter-domain rotation that accompanies arrestin activation, or how the active arrestin conformation initiates signaling. Finally, the arrestin-3 isoform is uniquely able to signal independently of GPCRs ${ }^{14-18}$. Previous studies identified that receptor-independent arrestin-3 activation biases signaling toward the activation of c-Jun N-terminal Kinase3 (JNK3) $^{14,16}$. However it is neither clear how arrestin-3 is activated in the absence of GPCRs nor are there compelling hypotheses for why receptor-independent arrestin-3 activation preferentially initiates the JNK3 signaling cascade. Indeed, it is not clear how arrestin activated by any input correctly directs signaling toward one out of $>100$ downstream effectors.

To address these questions, we determined the $2.4 \AA$ resolution crystal structure of arrestin-3 in complex with the non-receptor activator inositol hexakisphosphate $\left(\mathrm{IP}_{6}\right)$. We demonstrate that the same sensors critical for detecting the phosphorylated and activated state of GPCRs are triggered during receptorindependent activation, and reveal how the triggering of these sensors elicits inter-domain rearrangements. Moreover, we identify properties of the arrestin activation sensor that allow broad specificity for GPCRs. Finally, we propose a mechanism for arrestin-dependent signal initiation and bias. Collectively, these findings address many outstanding questions in the arrestin field.

\section{Results}

Structure of $\mathbf{I P}_{\mathbf{6}}$-activated arrestin-3. GPCR-independent arrestin-3 initiation of the JNK3 cascade is established in the literature ${ }^{14-18}$. Although a physiological non-receptor activator of arrestin-3 has not been unambiguously identified, inositol phosphates and heparin have been suggested as possibilities ${ }^{19}$. To test whether $\mathrm{IP}_{6}$ could activate arrestin-3, we determined the structure to $2.4 \AA$ resolution (Fig. 1a; Table 1), which shows that the $\mathrm{IP}_{6}$-arrestin-3 complex is a trimer (Fig. 1a). In the structure, we identified electron density consistent with two bound $\mathrm{IP}_{6}$ molecules per arrestin (Supplementary Fig. 1a, b); each $\mathrm{IP}_{6}$ molecule is located between the protomers of the arrestin trimer (Fig. 1a). The structure shows that $\mathrm{IP}_{6}$ binds to arrestin- 3 at the same location as $\mathrm{C}$-tail in basal arrestin such that $\mathrm{IP}_{6}$ displaces the C-tail (Supplementary Fig. 1c). The $\mathrm{IP}_{6}$-bound structure exhibits an inter-domain twist of $17.7^{\circ}$ with respect to the basal conformation (Fig. 1b). Moreover, the $\mathrm{IP}_{6}$ binding sites in the $\mathrm{N}$-domain overlap with the binding sites for phosphorylated receptor (Fig. 1c, d). These observations are consistent with this structure representing activated arrestin-3.

IP $_{6}$ binding supports GPCR-independent activation in cells. As $\mathrm{IP}_{6}$-bound arrestin-3 adopts an active conformation, we tested whether $\mathrm{IP}_{6}$ binding enhances effector binding in vitro and induces arrestin-3-dependent JNK3 activation ${ }^{14-18}$ in cells. To identify whether $\mathrm{IP}_{6}$ enhances JNK3 binding, we compared the capacity of column-immobilized $\mathrm{His}_{6}$-JNK3 to bind arrestin-3 in the presence or absence of $\mathrm{IP}_{6}$ (Fig. 1e). Even in the absence of $\mathrm{IP}_{6}$, arrestin-3 binds to JNK3, as described in ${ }^{17,20}$. This may reflect either low-affinity binding of JNK3 to the basal arrestin-3, or the ability of arrestin-3 to spontaneously sample the active conformation in the absence of activator. We observed an approximate doubling of the arrestin-3 binding to JNK3 in the presence of $\mathrm{IP}_{6}$ (Fig. 1e). This is consistent with $\mathrm{IP}_{6}$ shifting the conformational equilibrium of arrestin-3 to an active state that binds JNK3.

We next mutagenized the $\mathrm{IP}_{6}$ binding residues in the $\mathrm{N}$-domain $\left(\Delta \mathrm{N}_{\mathrm{IP} 6}\right.$; Fig. $\left.1 \mathrm{c}\right)$ or the C-domain $\left(\Delta \mathrm{C}_{\mathrm{IP} 6}\right.$, Supplementary Fig. 1d), then tested the impact on receptor-independent JNK3 activation in HEK293 cells (Fig. 1f). Co-expression of wild-type arrestin-3 with ASK1 (an upstream kinase in the JNK3 cascade $^{21}$ ) and JNK3 resulted in robust JNK3 phosphorylation. This was significantly attenuated in cells expressing the $\Delta \mathrm{N}_{\text {IP6 }}$ or $\Delta \mathrm{C}_{\mathrm{IP6}}$ arrestin-3 variants, providing strong evidence that $\mathrm{IP}_{6}$ activates arrestin-3 in cells, and facilitates arrestin-dependent JNK3 activation.

To rule out alternative interpretations for the loss of function in the arrestin-3 variants, we performed two types of assays. First, we tested whether the affinity of arrestin-3 for $\mathrm{IP}_{6}$ would allow binding at physiological concentrations of $\mathrm{IP}_{6}$. Second, we ensured that the $\Delta \mathrm{N}_{\text {IP6 }}$ and $\Delta \mathrm{C}_{\mathrm{IP6}}$ variants specifically reduced 


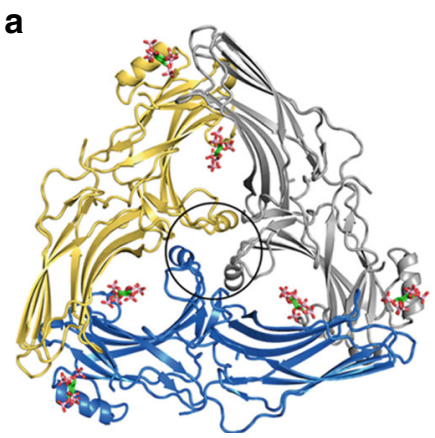

C

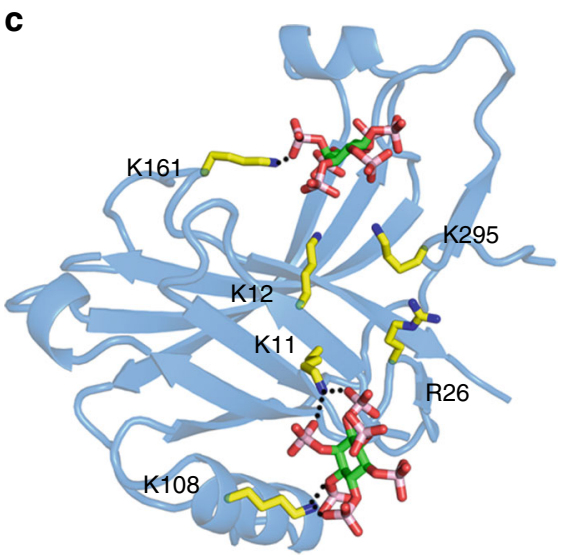

e

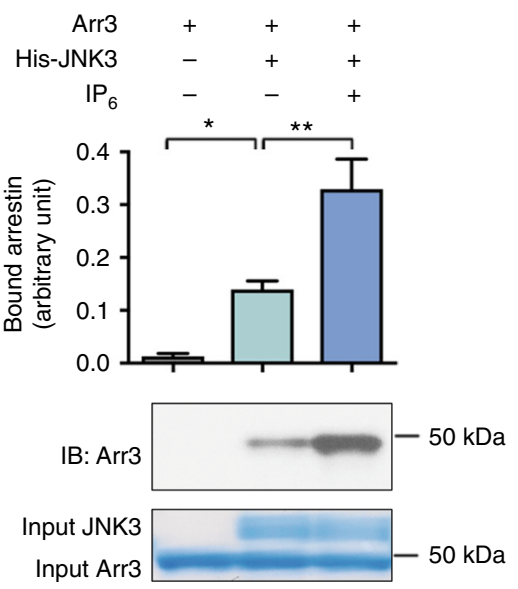

b

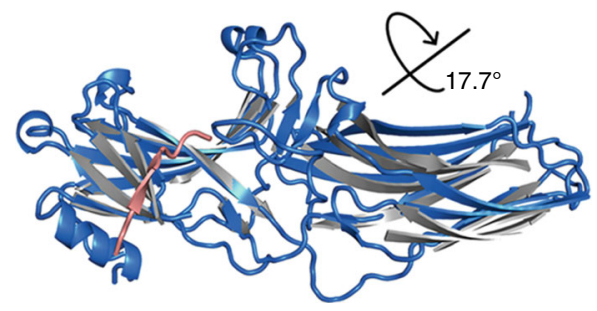

d

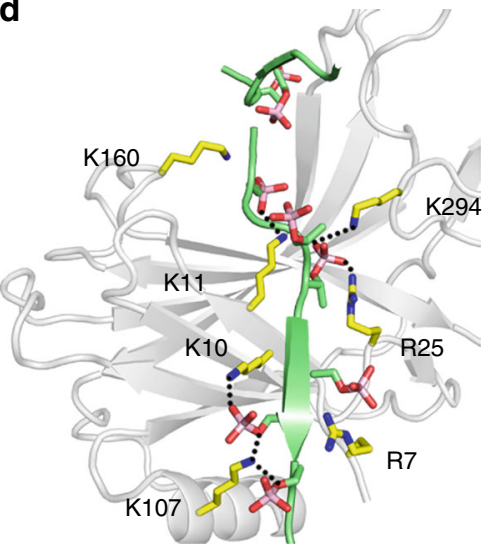

f
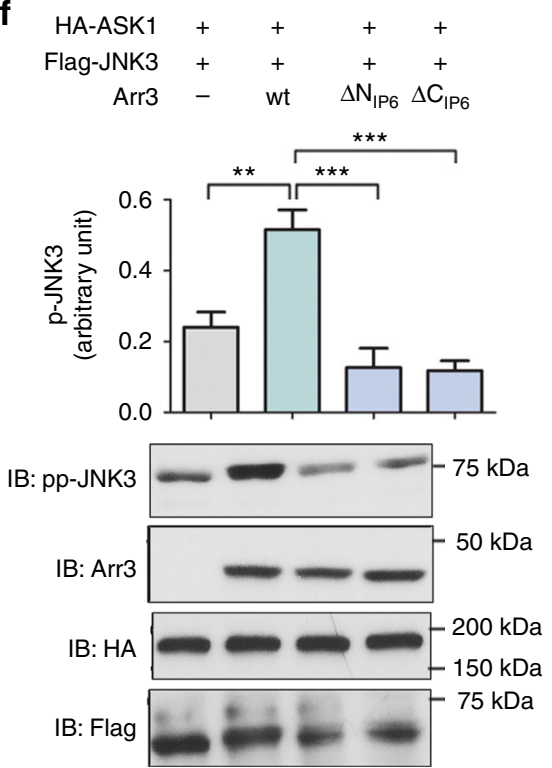

Fig. 1 The structure of $\mathrm{IP}_{6}$-activated arrestin-3. a Ribbon diagram of the $\mathrm{IP}_{6}$-activated arrestin-3 (Arr3) trimer. Each protomer is shown in a different color (gray, yellow, blue); $\mathrm{IP}_{6}$ is shown as sticks. Finger loops are circled. b Overlay of the $\mathrm{N}$-domain of $\mathrm{IP}_{6}$-activated (blue) with basal arrestin-3 (gray with a pink C-tail, PDB 3P2 ${ }^{6}$ ) highlights the $17.7^{\circ}$ inter-domain rotation. $\mathbf{c} \mathrm{IP}_{6}$ binding sites within the phosphate sensor on the arrestin-3 $\mathrm{N}$-domain. $\mathbf{d}$ Phosphopeptide binding sites within the phosphate sensor on arrestin-2 N-domain (PDB entry $4 \mathrm{JQI}^{17}$ ). e Arrestin-3 binding to purified His ${ }_{6}-\mathrm{JNK} 3 \mathrm{immobilized} \mathrm{on}$ $\mathrm{Ni}$-NTA resin in the presence or absence of $100 \mu \mathrm{M} \mathrm{IP} 6(n=8)$. The quantity of bound arrestin-3 was measured by densitometry (mean \pm SEM) and compared using one-way ANOVA. ${ }^{\star} p \leq 0.05 ;{ }^{\star \star} p \leq 0.01$. f Measurement of JNK3 activation by arrestin-3 and mutants of the IP 6 binding sites. JNK3 activation (mean \pm SEM) was assessed by measuring the phospho-JNK3 (ppJNK3) level in HEK293 cells co-transfected with HA-ASK1, Flag-JNK3 and either empty vector, arrestin-3, the $\Delta \mathrm{N}_{\mathrm{IP6}}$ arrestin-3 (R8Q, K11A, K12A, K108Q, K161Q, K295Q) or the $\Delta \mathrm{C}_{\mathrm{IP} 6}$ arrestin-3 (K233Q, R237Q, K251Q, K325Q, K327Q). The assay was repeated three times and JNK3 phosphorylation was compared using one-way ANOVA. ${ }^{\star \star} p \leq 0.01,{ }^{\star \star \star} p \leq 0.001$

receptor-independent signaling and had no global signaling defect.

To determine whether arrestin-3 binds $\mathrm{IP}_{6}$ at the concentrations found in cells, we used microscale thermophoresis (MST) to measure binding affinity. We labeled wild-type arrestin-3 with
AlexaFluor $\mathrm{C} 5$ maleimide dye, titrated $\mathrm{IP}_{6}$ into fluorophorelabeled arrestin-3, activated with an infrared laser, and monitored the fluorescence intensity. MST allowed us to obtain binding curves and calculate equilibrium dissociation constants. Wild-type arrestin-3 exhibits two binding affinities for $\mathrm{IP}_{6}\left(\mathrm{~K}_{\mathrm{D}}=57 \mathrm{nM}\right.$ and 
a

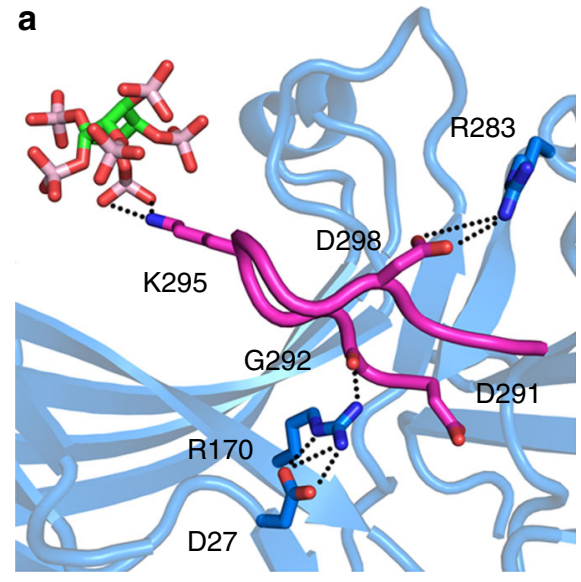

C
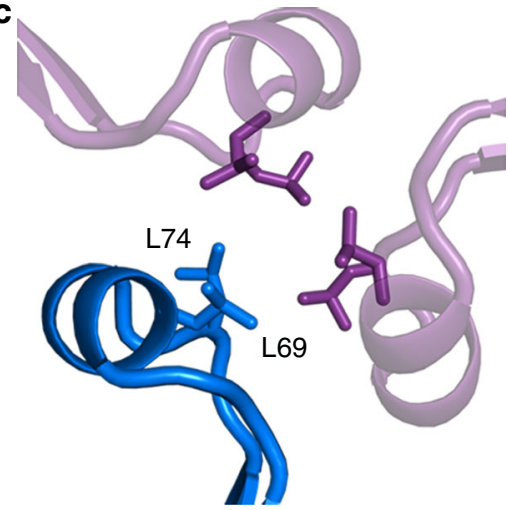

b
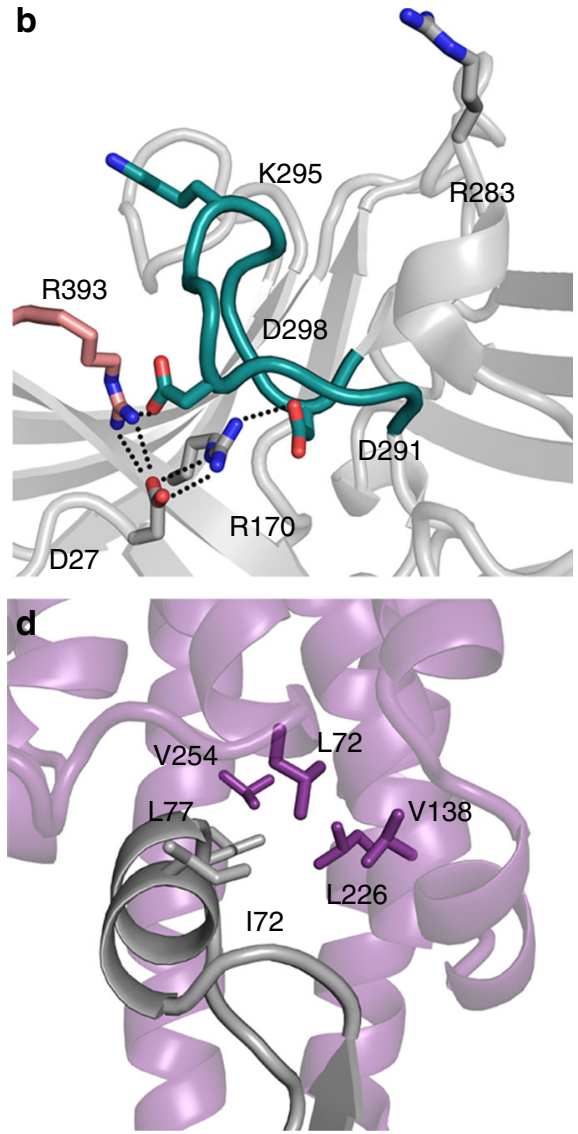

Fig. 2 The phosphate and activation sensors in $\mathrm{IP}_{6}$-activated arrestin-3. a Conformation of the lariat loop in $\mathrm{IP}_{6}$-activated arrestin-3 disrupts the polar core. b Conformation of the lariat loop in basal arrestin-3 (PDB 3P2D ${ }^{6}$ ) with an intact polar core. $\mathbf{c}$ Helical conformation of the finger loop stabilized by the active arrestin-3 trimer. d Similar hydrophobic contacts are observed in the arrestin-1-rhodopsin interaction (4ZWJ'13), and have distances of 3.5-4 $\AA$ between hydrophobic side chains

$90 \mu \mathrm{M}$; Supplementary Fig. 1e). As intracellular $\mathrm{IP}_{6}$ concentrations range between 35 and $105 \mu \mathrm{M}^{22}, 23$, these affinities are consistent with $\mathrm{IP}_{6}$ being able to occupy both binding sites in cells.

To ensure that that the mutants had no global signaling deficit, we evaluated folding and rhodopsin binding of purified wild-type and mutant arrestin- 3 in vitro. As the $\mathrm{IP}_{6}$ binding sites in the arrestin-3 $\mathrm{N}$-domain overlap with the binding sites for phosphorylated receptor (Fig. 1c, d), we anticipated that the $\Delta \mathrm{N}_{\text {IP6 }}$ variant would exhibit dramatically reduced receptor binding. However, the binding sites in the C-domain are unique to $\mathrm{IP}_{6}$, and if this variant is correctly folded and functional, the $\Delta \mathrm{C}_{\mathrm{IP} 6}$ mutations should only have a minor impact on receptor association. As anticipated, the $\Delta \mathrm{C}_{\mathrm{IP}}$ arrestin-3 variant retained significant receptor binding, while $\Delta \mathrm{N}_{\mathrm{IP} 6}$ arrestin-3 mutant showed a substantial reduction (Supplementary Fig. 1f) but was properly folded. Collectively, these experiments support the importance of the $\mathrm{IP}_{6}$-binding sites in receptor-independent arrestin-3 signaling.

$\mathrm{IP}_{6}$ triggers the arrestin-3 phosphate sensor. The $\mathrm{IP}_{6}$ molecules make extensive contacts with arginine and lysine side chains on the arrestin-3 N-domain (Fig. 1c). Critically, phosphate binding recruits Lys295 of the lariat loop, which contains two out of five charged residues in the polar core (Fig. 2a, b). This interaction likely elicits a conformational change that disrupts the polar core and triggers the phosphate sensor (Fig. 2a, b). A comparison of the $\mathrm{IP}_{6}$-bound structure to the reported structure of arrestin-2 with a vasopressin receptor phosphopeptide shows that the positions of the $\mathrm{IP}_{6}$ phosphates closely resemble those of the peptide-attached phosphates (Fig. 1c, d) ${ }^{11}$. The structure of arrestin- 3 in the basal conformation ${ }^{6}$ shows that the C-tail binds to the $\mathrm{N}$-domain, sterically occluding the phosphate binding sites (Supplementary Fig. 1c), but does not recruit Lys295. As a result, the polar core remains intact (Fig. 2b). Thus, $\mathrm{IP}_{6}$ and polyphosphated receptors bind to the same arrestin elements and trigger the phosphate sensor via the same mechanism by: (1) recruiting phosphate-binding side chains, (2) altering the conformation of the lariat loop, and (3) disrupting the polar core (Fig. 2a, b). This helps explain how relatively disparate phosphorylated species promote arrestin activation and helps clarify how the C-tail stabilizes the basal conformation.

Intriguingly, the Benovic and Brenner groups showed that soaking crystals of arrestin-2 with $\mathrm{IP}_{6}$ did not trigger the phosphate sensor ${ }^{24}$. Comparing the binding locations of the $\mathrm{IP}_{6}$ in arrestin- 2 vs. arrestin- $3^{24}$ shows that one of the $\mathrm{IP}_{6}$ molecules binds arrestin-2 in a different location (Fig. 1c, Supplementary Fig. 1d, g, h). As a result, $\mathrm{IP}_{6}$ binding to the arrestin-2 does not recruit Lys294 (equivalent to arrestin-3 Lys295). This leaves the polar core intact despite strict conservation of phosphate binding residues. While it is not clear how $\mathrm{IP}_{6}$ binding is directed differently in these isoforms, it is consistent with reports that arrestin-2 does not support receptor-independent signaling ${ }^{15,16,21}$.

Engagement of the arrestin-3 activation sensor. We wanted to explore whether the activation sensor is also triggered in $\mathrm{IP}_{6}$ activated arrestin-3. The activation sensor of arrestins is proposed to distinguish between active and inactive GPCRs ${ }^{5}$, although its 

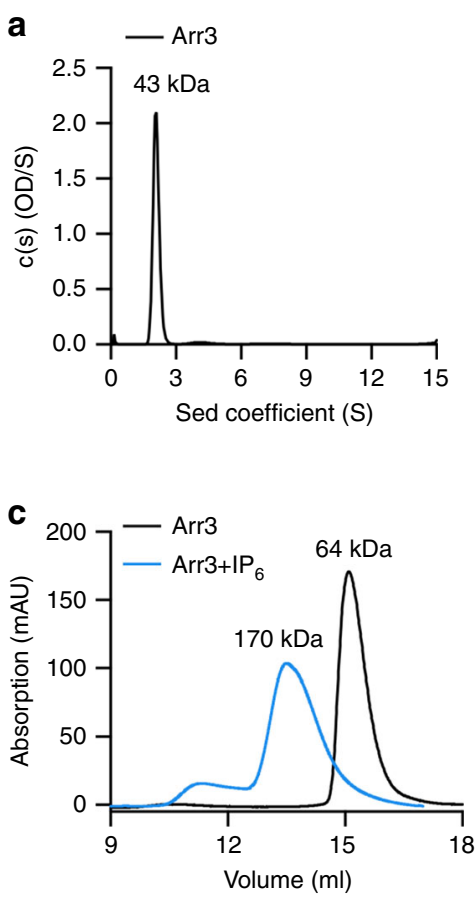

b
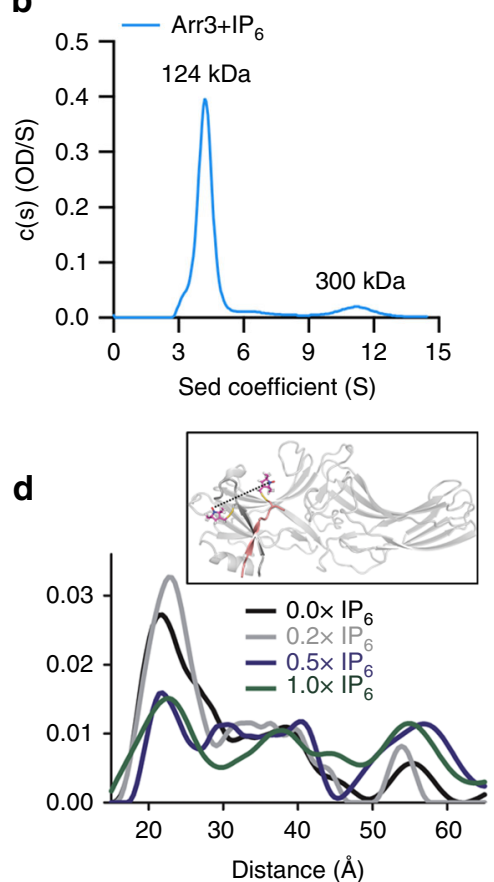

e

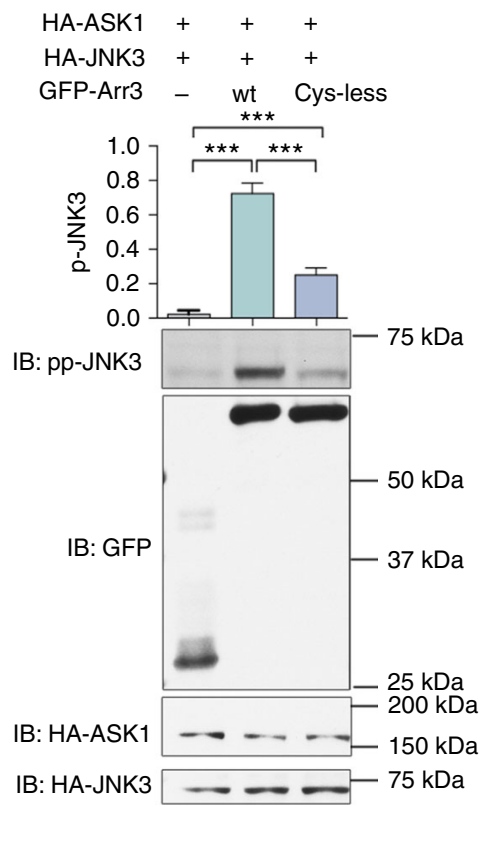

Fig. $3 \mathrm{IP}_{6}$ mediated trimerization and receptor-independent activation in cells. a-b Sedimentation velocity analytical ultracentrifugation (SVAUC) of arrestin-3. Measurements were performed in triplicate. a Representative SVAUC run in the absence of $\mathrm{IP}_{6}$ predicts a molecular weight matching a monomer. $\mathbf{b}$ In the presence of $100 \mu \mathrm{M} \mathrm{IP}_{6}$, the predicted molecular weight is consistent with a trimer. $\mathbf{c}$ Representative size exclusion chromatograms of arrestin-3 (Arr3) in the presence and absence of $\mathrm{IP}_{6}$. In the absence of $\mathrm{IP}_{6}, 60 \mu \mathrm{M}$ arrestin-3 (black) elutes at volume corresponding to the Stokes radius of a globular protein with a molecular mass of $64 \pm 5.9 \mathrm{kDa}$. In the presence of $100 \mu \mathrm{M} \mathrm{IP}_{6}$, the elution volume is consistent with a globular protein of molecular mass $169.6 \pm 9.7 \mathrm{kDa}$. The ratio of these molecular weights is consistent with $\mathrm{IP}_{6}$-dependent trimer formation. Measurements were performed in triplicate, errors are \pm SEM. d Plot of the probability of the distances between spin labels at S13 and A392 for $100 \mu \mathrm{M}$ arrestin-3 in the presence of the indicated molar ratios of $\mathrm{IP}_{6}$; inset shows the location of the spin labeled sites in basal arrestin-3 (PDB entry 3P2D ${ }^{6}$ ). e Comparison of JNK3 activation by GFP-arrestin-3 and Cys-less mutant. GFP-tagged arrestin allowed comparison of the expression levels of wild-type and variant arrestin-3, as described ${ }^{14,33}$. JNK3 activation (mean \pm SEM) was assessed by measuring the pp-JNK3 levels in COS7 cells co-transfected with HA-ASK1, HA-JNK3 and GFP or Venustagged wild-type and Cys-less arrestin-3. Assay was repeated five times and JNK3 phosphorylation was compared by one-way ANOVA followed by Bonferroni post hoc test with correction for multiple comparisons. ${ }^{\star \star \star} p<0.001$

identity has not been suggested in the literature. To offer insight into the identity of the activation sensor, we analyzed differences between active and inactive receptors. The most striking structural difference is the accessibility of a hydrophobic pocket on the intracellular side of the protein unique to active GPCRs ${ }^{25}$. This pocket represents a major site of interaction with both $G$ proteins $^{26,27}$ and arrestins ${ }^{13,28}$. In fact, it contributes $596 \AA^{2}$ out of a total of $1362 \AA^{2}$ of buried surface area in the rhodopsin-arrestin complex ${ }^{15}$. Cocrystal structures of opsin with pre-activated arrestin-1 or an arrestin-derived peptide suggest that the hydrophobic pocket binds to an $\alpha$-helical conformation of the finger loop ${ }^{13}$, 28 . However, there is controversy on this because of the quality of the electron density for this helix in these structures (Supplementary Fig. 2a, b). The $\alpha$-helix of the finger loop contains a $\mathrm{ED}(\mathrm{I} / \mathrm{L}) \mathrm{D}$ motif (residues $68-71$ of arrestin-3) ${ }^{13}, 28$ that lacks secondary structure in basal arrestins $s^{4,6-8}$ and is not altered in partially activated arrestin ${ }^{29}$. Helix formation of the finger loop presents several hydrophobic residues to the intracellular hydrophobic pocket of activated receptor ${ }^{13,28}$.

Similarly, in the $\mathrm{IP}_{6}$-mediated trimer the finger loop is presented as an $\alpha$-helix (Fig. 2c, d). In contrast with the receptor-bound structure ${ }^{13}$, this helix is associated with clear electron density (Supplementary Fig. 2c). In this conformation, hydrophobic residues would be exposed to solvent if the $\mathrm{IP}_{6^{-}}$ activated arrestin-3 were a monomer, but in the context of the trimer, these form Van der Waals $(<4 \AA)$ self-contacts around the molecular three-fold axis (Fig. 2c) and are shielded from solvent.
This suggests that receptor-independent trimerization and the GPCR hydrophobic pocket stabilize the triggered activation sensor in the same way (Fig. $2 c, d$ ).

IP $_{6}$-mediated arrestin-3 trimerization and activation. If trimerization stabilizes the triggered activation sensor, then the trimer should be quite stable in the presence of $\mathrm{IP}_{6}$. It would also be critical for receptor-independent activation of arrestin-3. Importantly, the $\mathrm{IP}_{6}$-bound arrestin- 3 trimer buries $3053 \AA^{2}$ of surface area per protomer, a value indicative of a biologically relevant oligomer ${ }^{30}$. The trimer does not appear to be influenced by crystal contacts (Supplementary Fig. 3a); however, it does require the inter-domain twist of active arrestin. Indeed, modeling the basal conformation causes the misalignment of the $\mathrm{IP}_{6}$ binding sites (Supplementary Fig. 3b). This would be predicted to reduce $\mathrm{IP}_{6}$ affinity and therefore disfavor trimerization. We therefore next explored the stability of the arrestin- 3 trimer in solution.

Using analytical ultracentrifugation, we found that the addition of $\mathrm{IP}_{6}$ to purified arrestin-3 converts the observed molar mass from $43 \pm 1 \mathrm{kD}$ (monomer) to $134 \pm 5 \mathrm{kD}$ (trimer) (Fig. 3a, b). Size exclusion chromatography (Fig. 3c, Supplementary Table 1) is also consistent with $\mathrm{IP}_{6}$-dependent trimerization of arrestin-3. We then explored the effect of arrestin- 3 concentration on $\mathrm{IP}_{6}$ dependent trimerization and found that the trimer is stable at the lowest arrestin-3 concentration detectable by our instruments $(\sim 1 \mu \mathrm{M}$; Supplementary Fig. $3 c)$. 
a

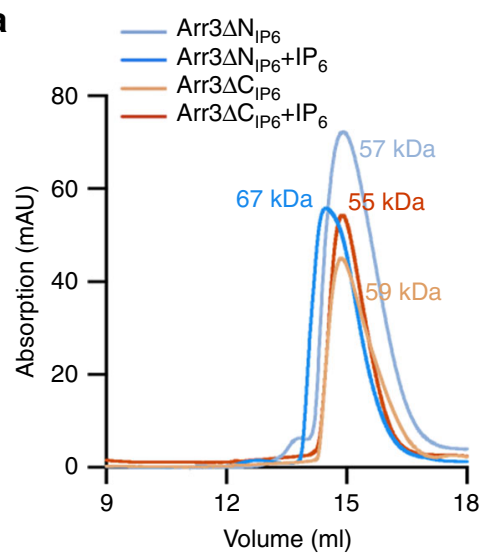

c

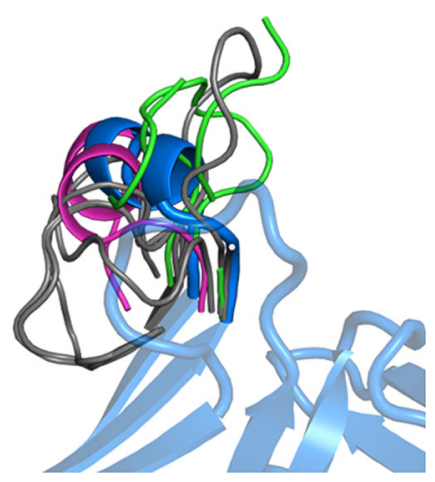

e

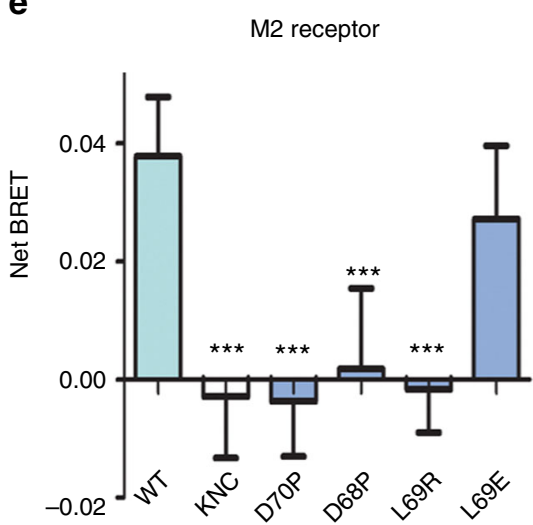

b

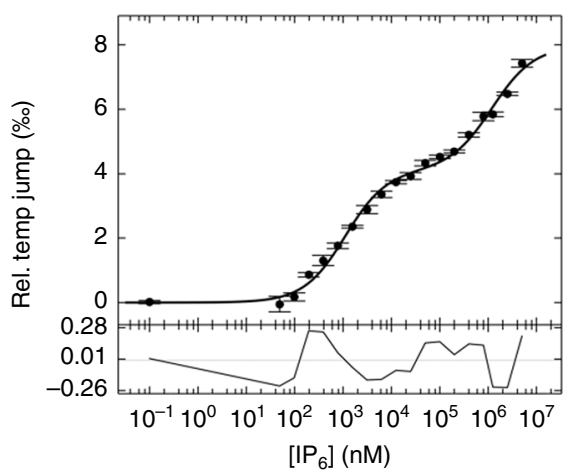

d

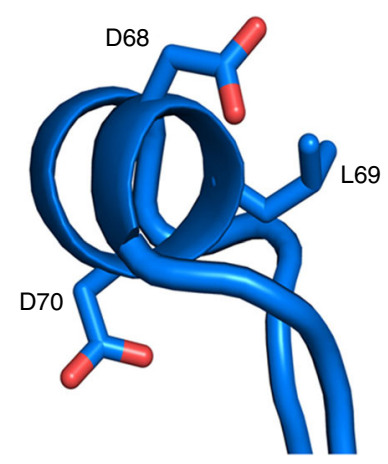

f

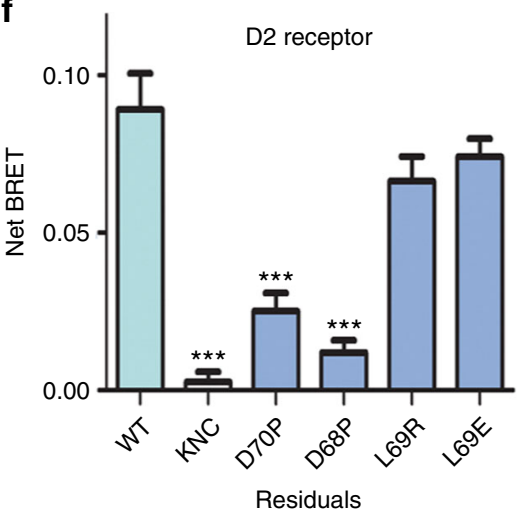

Fig. 4 The interplay between phosphate and activation sensors in receptor-independent and receptor-dependent signaling. a Size exclusion chromatography (SEC) of the $\Delta \mathrm{N}_{\mathbb{I P 6}}$ and $\Delta \mathrm{C}_{\mathbb{P P} 6}$ mutants measured on a Superdex S200 Increase 10/300 GL column (24 mL). Arrestin-3 (1-393) runs anomalously on size exclusion chromatography, but exhibits a characteristic shift in molecular weight upon the addition of $I P_{6}$. In the absence of $I P_{6}$, both the $\Delta \mathrm{N}_{\mathrm{IPG}}$ and the $\Delta \mathrm{C}_{\mathrm{IP} 6}$ mutants are monodisperse and have a similar elution volume to wild-type, but in the presence of $\mathbb{I}_{6}$, no mobility shift is observed. b Temperature-jump binding curve for the Cys-less-T222C arrestin-3. c Overlay of finger loop of arrestin structures. Basal (gray): PDB entries 1CF1 ${ }^{7}$, $1 \mathrm{JSY}{ }^{35}, 1 \mathrm{ZSH}^{24}, 3 \mathrm{P} 2 \mathrm{D}^{6}, 1 \mathrm{G} 4 \mathrm{M}^{4}$; active (green): PDB entries $4 \mathrm{ZRG}{ }^{12}, 4 \mathrm{JQ} \mathrm{I}^{11}, 4 \mathrm{~J} 2 \mathrm{Q}^{10}$. Bound to receptor (magenta): $4 \mathrm{ZWJ} \mathrm{J}^{13}$. Bound to IP 6 (blue) $\mathbf{d} \mathrm{The}$ conserved motif $\mathrm{EDL} /(\mathrm{I}) \mathrm{D}$ folds into an $\alpha$-helix. e, f Evaluation of mean binding $\pm \mathrm{SEM}$ of wild-type and mutant Venus-arrestin-3 binding to the luciferasetagged e M2 muscarinic or $\mathbf{f}$ D2 dopamine receptor by BRET. In arrestin-3-KNC (K11A, K12A, L49A, D51A, R52A, L69A, Y239A, D241A, C252A, P253A, D260A and Q262A), two key phosphate-binding lysines and 10 residues that bind other parts of the receptor were mutated to alanines. This precludes GPCR binding as described ${ }^{22,34}$, making this an appropriate negative control. Data from three experiments were compared to wild-type by one-way ANOVA. ${ }^{\star \star \star} p \leq 0.001$

We next assessed whether trimerization contributes to receptor-independent activation in cells using a trimerization deficient arrestin-3 variant. To design a trimerization deficient variant, we identified residues that are surface exposed in basal (monomeric) arrestin-3, buried in the trimer interface, and not associated with known biochemical functions of arrestin. This suggested Cys17 as a candidate side chain for targeting. We used a fully Cys-less variant of arrestin-3 because it has previously been shown to bind receptors normally ${ }^{31}$. This indicates that the Cys-less variant is folded and rules out many alternative interpretations of any results.

We first assessed whether the Cys-less variant lost the ability to form trimers, using size exclusion chromatography. We found that the Cys-less variant does not form stable trimers in response to $\mathrm{IP}_{6}$, but appears to shift to a molecular weight consistent with a dimer (Supplementary Fig. 3d). Comparing the arrestin-3 trimer 
to the arrestin-2 oligomers suggests that while the trimer can stabilize the inter-domain twist (Fig. 1a, b), other arrestin oligomers cannot. This makes Cys-less arrestin-3 suitable for measuring how trimerization impacts receptor-independent signaling.

To quantify $\mathrm{IP}_{6}$-dependent activation of Cys-less arrestin-3 in vitro, we employed double electron electron resonance (DEER) spectroscopy. One of the hallmarks of arrestin activation is the displacement of the $\mathrm{C}$-tail from the $\mathrm{N}$-domain upon the binding of phosphates to the phosphate sensor. We monitored this process via attached spin labels to cysteines replacing Ser13 in the $\mathrm{N}$-domain and Ala392 in the C-tail. At these positions, the spin labels are separated by $22 \AA$ in basal arrestin-3, when the C-tail is bound to the $\mathrm{N}$-domain. Upon arrestin activation and $\mathrm{C}$-tail release, these convert to a wide distribution of longer inter-spin distances. After titration with $\mathrm{IP}_{6}$, we found that only $~ 30 \%$ of the $22 \AA$ distance converted to longer distances (Fig. 3d, Supplementary Fig. $3 \mathrm{e}$ ), $\mathrm{a} \sim 70 \%$ loss in $\mathrm{IP}_{6}$-dependent activation. Cys-less arrestin-3 was reported to exhibit nearly $100 \%$ release of the C-tail in response to phosphorhodopsin in the same assay ${ }^{31}$, which rules out the possibility that Cys-less arrestin-3 is signaling deficient.

We then compared the ability of wild-type and Cys-less arrestin-3 to mediate receptor-independent JNK3 activation in cells. We observed a $65 \%$ reduction in JNK3 activation in cells expressing the Cys-less mutant as compared to wild-type arrestin3 (Fig. 3e). This correlates with the loss of $\mathrm{IP}_{6}$-dependent activation measured by DEER.

Our observed trimer contrasts with reported multi angle laser light scattering (MALLS) of $\mathrm{IP}_{6}$-bound arrestin-3, which were explained by a dimer ${ }^{32}$. To investigate this discrepancy, we evaluated differences in the experimental design and identified that a protease inhibitor, benzamidine, used in the MALLS studies induces heterogeneous oligomerization (Supplementary Fig. 4a-c, details in the legend), with oligomers having an average molecular weight consistent with a dimer. As benzamidine is not present in cells, it is likely that these oligomers are non-physiological.

The phosphate and activation sensors are intimately linked. With the phosphates of $\mathrm{IP}_{6}$ apparently triggering the phosphate sensor and trimerization likely triggering the activation sensor, we wanted to explore the relationship between $\mathrm{IP}_{6}$-binding and trimerization. Since $\mathrm{IP}_{6}$ molecules mediate trimer formation, we propose that $\mathrm{IP}_{6}$-binding and trimerization are intertwined. This would suggest that triggering the phosphate and activation sensors are linked during receptor-independent arrestin-3 activation.

To test this, we first evaluated the ability of the $\Delta \mathrm{N}_{\text {IP6 }}$ and $\Delta \mathrm{C}_{\mathrm{IP} 6}$ variants of arrestin-3 to form $\mathrm{IP}_{6}$-dependent trimers. As described above, the $\mathrm{IP}_{6}$-binding sites are altered by mutagenesis in these variants, and receptor-independent JNK3 activation in cells is compromised (Fig. 1f). Size exclusion chromatography showed that these variants no longer trimerize in the presence of $\mathrm{IP}_{6}$, instead exhibiting mobility consistent with a monomer (Fig. 4a, Supplementary Table 1).

We next tested how loss of trimerization impacts $\mathrm{IP}_{6}$ affinity using MST. The Cys-less variant is a valuable tool for this measurement because trimerization is disrupted; however, our experimental design used a maleamide-conjugated fluorophore, which interacts with cysteines. Thus, we re-introduced a cysteine residue to allow labeling. The T222C variant was considered suitable for this purpose because T222 is distal from all characterized functional regions. This mutant is also monodisperse, consistent with correct folding (Supplementary
Fig. 3c). When compared to wild-type arrestin-3 $\left(K_{D}=57 n M\right.$ and $90 \mu \mathrm{M}$; Supplementary Fig. 1e), the T222C-Cys-less variant shows substantially reduced $\mathrm{IP}_{6}$ binding affinity $\left(\mathrm{K}_{\mathrm{D}}=1.1 \mu \mathrm{M}\right.$ and $1.2 \mathrm{mM}$, Fig. 4b), consistent with trimerization contributing to $\mathrm{IP}_{6}$ affinity.

Collectively, these data strongly suggest $\mathrm{IP}_{6}$-binding and trimerization work together to support receptor-independent arrestin- 3 activation of JNK3. Because $\mathrm{IP}_{6}$ appears to directly bind the phosphate sensor, and trimerization appears to stabilize a triggered activation sensor, we conclude that during receptorindependent activation, triggering of these sensors is intimately linked. This mirrors the observed synergy of these two sensors in receptor-dependent signaling 5 .

Arrestin-3 sensors in broad receptor selectivity. There are $>800$ GPCRs, but only two non-visual arrestins (arrestin-2 and arrestin-3) that recognize the phosphorylated and activated forms of these receptors; arrestin-3 also recognizes non-receptor activators. Intuitively, the mechanism of broad receptor specificity of the phosphate sensor is straightforward, as the $\mathrm{N}$-domain of arrestin can bind receptor-attached phosphates with the correct spacing. In contrast, it is less clear how the activation sensor might recognize this large number of receptors.

If our assignment of the finger loop as a part of the activation sensor is correct, this element must interact with the hydrophobic pocket on $>800$ GPCRs with limited sequence similarity. We therefore analyzed the finger loop to identify properties that could contribute to broad receptor recognition. An overlay of available arrestin structures suggests that the finger loop is on a flexible tether (Fig. 4c) and can be presented at many angles to an interaction partner. This could allow the finger loop to adapt to the different hydrophobic pockets on receptors. If so, the helicity, hydrophobicity, and flexibility of the finger loop would be predicted to contribute to the broad receptor specificity of the activation sensor. Hence, perturbation of these properties would reduce activation sensor-dependent receptor binding. We therefore designed mutations (Fig. 4d) that introduced (a) a helixbreaking proline (D68P), (b) a flexibility-reducing proline terminating the helix (D70P), or (c) a charge within the hydrophobic region (L69E/R). Modeling based on available structures $^{13,28}$ suggests that neither introduced proline should directly interact with the receptor.

We measured the binding of these variants to M2 muscarinic and D2 dopamine receptors, which we selected because they are more dependent upon the activation sensor than on receptorattached phosphates for arrestin binding ${ }^{33}$. We used a bioluminescence resonance energy transfer (BRET) assay in COS7 cells cotransfected with luciferase-tagged receptors and Venus-tagged arrestin- $3^{14}$ (Fig. 4e, f, Supplementary Fig. 5a, b). Wild-type arrestin-3 shows a robust increase in BRET signal upon agonist stimulation, whereas arrestin-3-KNC (a negative control with 12 substitutions of key receptor-binding residues that binds poorly to receptors ${ }^{14}$ ) does not ${ }^{33,34}$. Mutants designed to perturb helix formation or flexibility had substantially reduced binding to both receptors (Fig. 4e, f). Interestingly, charged residues had different effects on binding to the M2 and D2 receptors. This variability may reflect the presence or absence of complementary charges in the inter-helical cavity of different GPCRs, or the strength of other interactions between receptor and arrestin-3.

As the finger loop is also a major contact for $\mathrm{IP}_{6}$-dependent arrestin-3 trimerization, we tested if finger loop variants affect receptor-independent activation and signaling. Alteration of the finger loop prevented $\mathrm{IP}_{6}$-dependent trimerization of the $\mathrm{D} 68 \mathrm{P}$ variant, reduced trimerization of the L69R and D70P mutations, and had no detectable impact in the L69E variant (Supplementary 


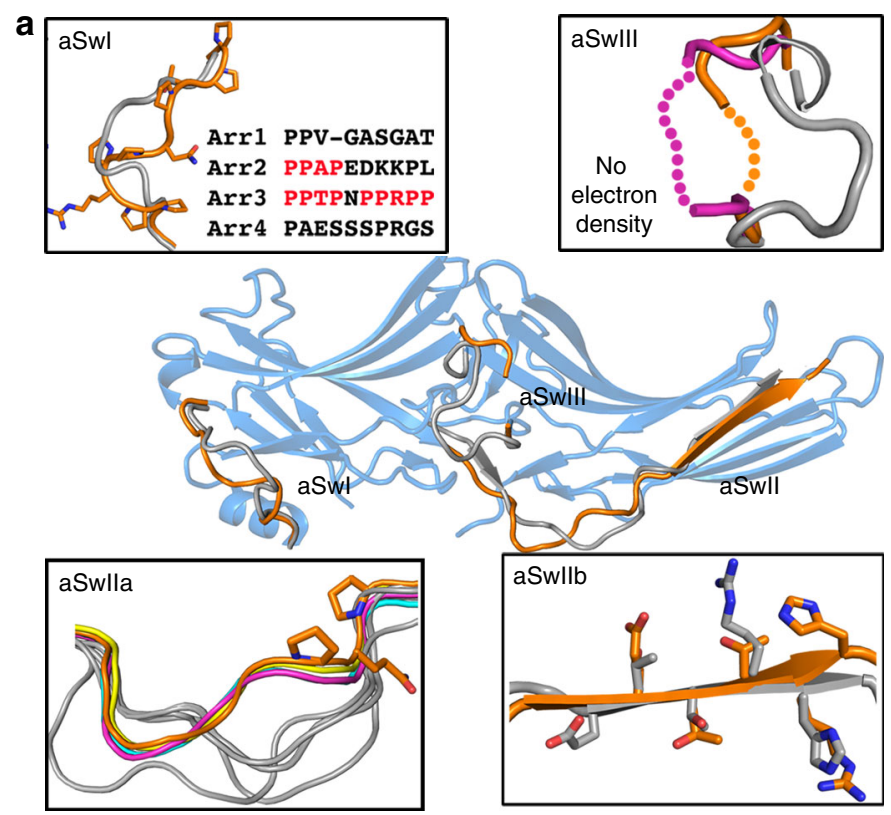

b

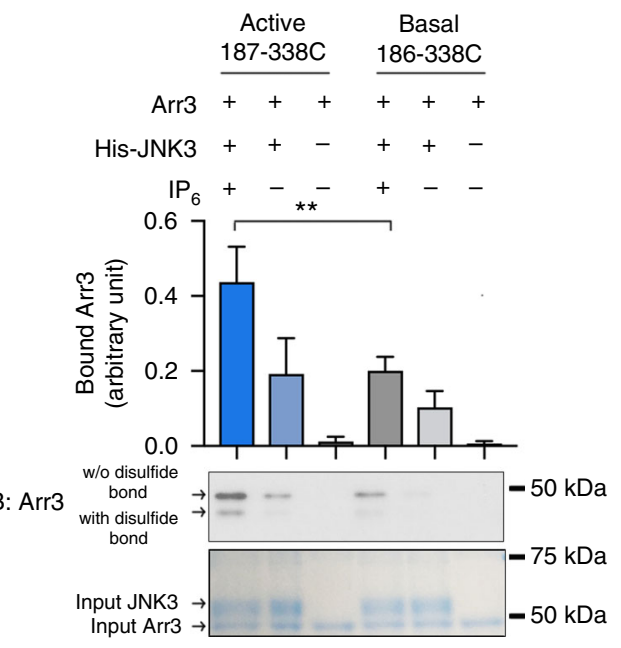

Fig. 5 Arrestin switch regions. a Conformations of the arrestin switch regions in the active form (orange ( $\mathbb{P}_{6}$-activated arrestin-3 (Arr3)), magenta (phosphopeptide-activated arrestin-2; PDB 4JQI ${ }^{11}$ ), cyan (rhodopsin-activated arrestin-1; (PDB 4ZWJ'13)), yellow (p44; PDB 4J2Q $\left.{ }^{10}\right)$ ) differ from those in basal arrestin-3 (gray, PDB 3P2D6). Insets highlight switch regions. In the inset showing aSwl, the sequences in four bovine arrestin isoforms are shown. b Quantitation of arrestin-3 binding to $\mathrm{His}_{6}-\mathrm{JNK} 3$ in the presence and absence of $\mathrm{IP}_{6}(100 \mu \mathrm{M})$, monitored using an in vitro pull-down assay. JNK3 was immobilized on $\mathrm{Ni}^{2+}$ resin, exposed to arrestin-3 (with disulfides stabilizing active or basal aSwllb), washed, then eluted in sample buffer and visualized by SDS-PAGE and Western. Disulfide trapped arrestin-3 (red box) was quantified separately by densitometry and the ratio was calculated (means \pm SEM). ${ }^{\star \star} p \leq 0.01$. The overall amount was normalized by the ratio of disulfide trapped arrestin (Supplementary Fig. 5)

Fig. 5c, d). JNK3 activation in cells was not affected in the D69R and D70P mutations (Supplementary Fig. 5e). One interpretation of this finding is that $\mathrm{IP}_{6}$-induced arrestin-3 activation is more dependent upon the phosphate sensor than the activation sensor, a property shared with many GPCRs ${ }^{33}$. However, given our data suggesting that triggering the phosphate and activation sensors during receptor-independent activation is closely linked, it is more likely that the finger loop of our variants is presented in a way that allows trimerization.

Switch regions in arrestin-mediated signaling. A major functional consequence of non-visual arrestin activation is the engagement of downstream effectors, yet how activated arrestin supports signaling has never been explained. In the $\mathrm{IP}_{6}$-activated arrestin-3 structure we identified structural changes in known effector binding elements that may promote signal initiation. Activation-induced conformational changes in these regions have not been previously reported, although inspection of the coordinates for the active arrestin-1 and arrestin-2 indicates that similar changes accompany activation of other arrestins ${ }^{10,11,13}$. We suggest that these conformational changes act as molecular switches functionally analogous to the switch regions of $G$ proteins [reviewed in 35], and therefore term these "arrestin switch" regions. In conjunction with the inter-domain twist, these conformational changes would create effector-binding sites and turn on signaling when arrestin is activated.

Arrestin switch I (aSwI; residues 89-97; Fig. 5a) is strongly conserved in arrestin-3 homologs from other species, but not in the other three arrestin isoforms (Fig. 5a, inset). In arrestin-3, this nine amino acid segment contains seven prolines, including two PPXP motifs that may be recognized by $\mathrm{SH} 3$ domains ${ }^{35}, 36$. Comparison of the basal ${ }^{6}$ and active conformations of aSwI reveals a maximal displacement of $5.8 \AA$ and includes an unusual pair of tandem cis bonds (Pro94-Pro95 and Pro95-Arg96) in the best-fitting model. This rare structural feature is associated with conformational change $e^{37,38}$. However, the electron density of this region is difficult to interpret, and not all residues could be modeled with confidence (Supplementary Fig. 6a). The flexibility of this switch may be important for adapting to different effectors.

Arrestin switch II (aSwII; residues 176-191; Fig. 5a) contains two distinct parts: IIa (residues 176-183) and IIb (184-191). ASwIIa connects the $\mathrm{N}$ - and $\mathrm{C}$-domain, and has been proposed to act as a hinge between domains ${ }^{39,40}$. ASwIIa also contains a polyproline motif (PQP residues 181-183) suggested as a non-canonical SH3 binding site in arrestin- $2^{35}$. The aSwIIa shows a nearly identical conformation in all available active arrestin structures, but variable conformations in basal arrestins (Fig. 5a, inset).

ASwIIb includes the entirety of the first $\beta$-strand of the C-domain (residues 184-191) and superimposes in all active arrestin structures. As compared to most arrestins in the basal state, the $\beta$-strand of aSwII is register-shifted, which moves it one position in arrestin-2 and -3 and two positions in arrestin-1 (Supplementary Fig. 6b, c), although the active position of aSwIIb is observed in one crystal form of basal arrestin-2 24,35 (Supplementary Fig. 6b). Register-shifted $\beta$-strands are unusual, but have proposed roles in regulating signaling ${ }^{41,42}$ and forming protein binding sites ${ }^{43}$.

Arrestin switch III (aSwIII, residues 307-316; Fig. 5a) is an extension of the lariat loop ${ }^{7}$, which is a part of the polar core that stabilizes the orientation of the two domains in the basal state. ASwIII becomes disordered in both activated arrestin-3 and arrestin- $2^{11}$, a property commonly associated with the ability to mediate protein-protein interactions ${ }^{44,45}$.

Using the same terminology, the arrestin C-tail may be considered aSwIV. In basal arrestins, the C-tail is anchored to the $\mathrm{N}$-domain ${ }^{4,6-8,35}$. Receptor binding induces release of the C-tail, which becomes unstructured $31,46,47$. The binding sites for clathrin ${ }^{48}$ and clathrin adapter $\mathrm{AP}-2^{48}$ are localized in the 
a

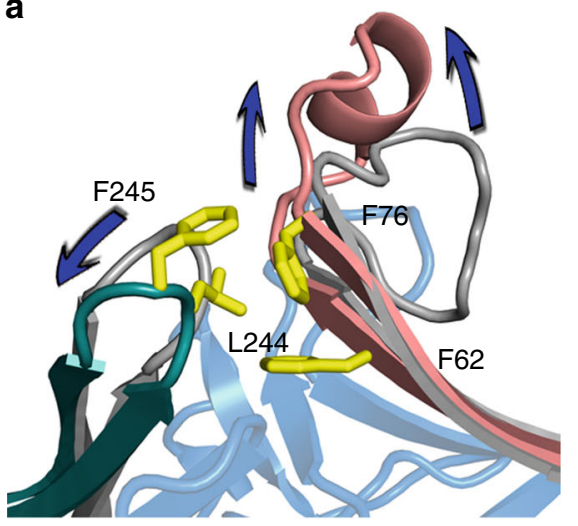

c

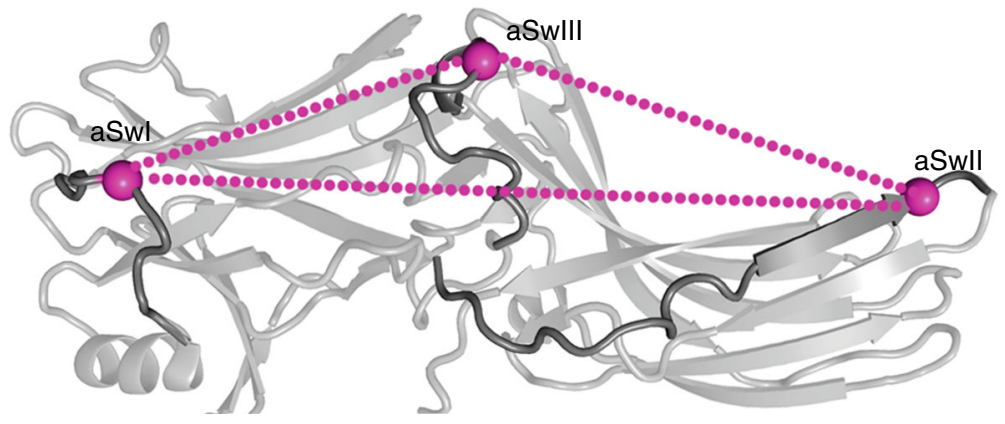

d

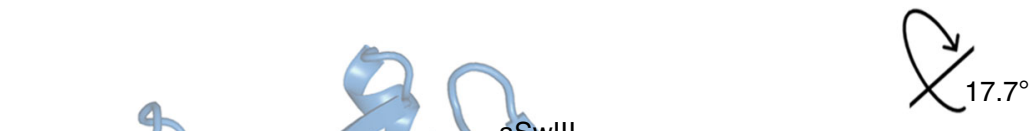

b $\quad$ Finger

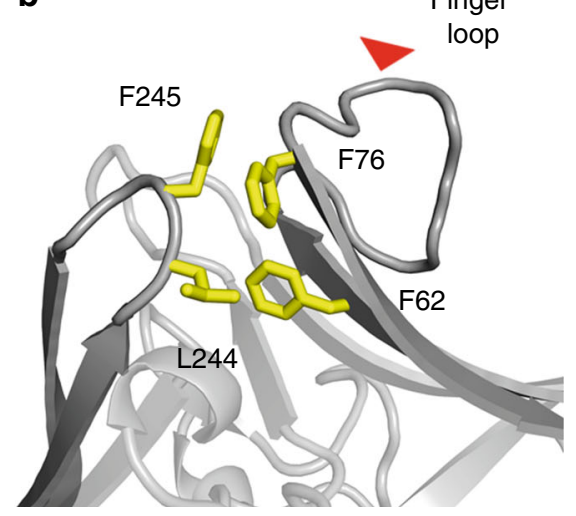




\begin{tabular}{|c|c|}
\hline \multicolumn{2}{|l|}{ Data collection } \\
\hline Beamline & LS-CAT ID-D \\
\hline Wavelength & $1.7394 \AA$ \\
\hline Resolution $(\AA)$ & $50.0-2.4$ \\
\hline Space group & $\mathrm{P}_{3}$ \\
\hline Unit cell dimensions & $a=b=97.575 \AA, c=76.938 \AA$ \\
\hline $\mathrm{R}_{\mathrm{sym}}$ & $0.051(0.546)$ \\
\hline$R_{\text {pim }}$ & $0.031(0.416)$ \\
\hline $\mathrm{CC}_{1 / 2}{ }^{\mathrm{b}}$ & 0.865 \\
\hline $\mathrm{I} / \sigma$ & $26.2(2.00)$ \\
\hline Completeness & $97.5(96.0)$ \\
\hline Redundancy & $3.4(2.6)$ \\
\hline \multicolumn{2}{|l|}{ Refinement } \\
\hline $\mathrm{R}_{\text {cryst }}{ }^{\mathrm{c}}$ & 0.210 \\
\hline$R_{\text {free }}$ & 0.243 \\
\hline \multicolumn{2}{|l|}{ RMS deviation from ideal } \\
\hline Bond lengths & $0.004 \AA$ \\
\hline Bond angles & $1.09^{\circ}$ \\
\hline \multicolumn{2}{|l|}{ Ramachandran statistics } \\
\hline Most favored & $95.0 \%$ \\
\hline Additionally allowed & $4.5 \%$ \\
\hline Disallowed & $0.6 \%$ \\
\hline
\end{tabular}

aNumbers in parenthesis are values for data in the outer resolution shell, corresponding to 2.44-2.40 A resolution

$2.44-2.40 \mathrm{~A}$ resolution
${ }^{\mathrm{b}} \mathrm{C}_{1 / 2}$ is only reported for the outer resolution shell

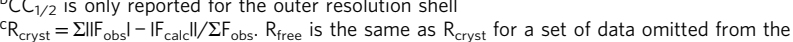
refinement

We then assessed the binding of each of these disulfidestabilized versions of arrestin-3 to JNK3. In the presence of $\mathrm{IP}_{6}$, column-immobilized JNK3 exhibits increased binding to arrestin3 with aSwII trapped in the active conformation (Fig. 5b). In the absence of $\mathrm{IP}_{6}$, the binding is lower for both variants, suggesting that the disulfide-trapped aSwII does not fully shift the arrestin equilibrium to an active state in the absence of $\mathrm{IP}_{6}$.

\section{Discussion}

We used $\mathrm{IP}_{6}$-bound arrestin-3 to identify the structural requirements for receptor-independent arrestin activation, and to suggest how activation results in arrestin-mediated signaling. These data inform on several aspects of arrestin activation that have been enigmatic: how an arrestin can interact with many disparate receptor and non-receptor activators, adopt an active conformation, then initiate a variety of downstream signaling pathways.

Arrestin-3 is activated by $>800$ GPCRs plus non-receptor activators, including $\mathrm{IP}_{6}$. When coupling to receptors, arrestin acts as a coincidence detector that binds with high affinity to phosphorylated and activated receptors, as mediated by two independent sensors ${ }^{5}$. We show that the $\mathrm{IP}_{6}$ triggers the same two sensors. Indeed, the binding site for $\mathrm{IP}_{6}$ sterically overlaps with the binding site for receptor-attached phosphates (Fig. 1c, d). Similarly, the surface of the finger loop that interacts with the hydrophobic pocket of receptor also interacts with sister protomers in the trimer ${ }^{13,28}$ (Fig. $2 \mathrm{c}, \mathrm{d}$ ). Because the same surfaces support receptor-independent trimerization and receptor binding, receptor-dependent and receptor-independent arrestin-3 activation appear to be mutually exclusive, i.e., the trimer does not bind receptors. The use of the same sensors and surfaces for trimerization and receptor binding also suggests that receptordependent and receptor-independent arrestin activation occur via a similar mechanism.

Structurally, arrestin activation involves several conformational rearrangements. First, negatively charged phosphates bind to the
$\mathrm{N}$-domain, displacing the $\mathrm{C}$-tail and disrupting the polar core ${ }^{11}$. Our data and previous reports suggest the disruption of the polar core by phosphate binding is a hallmark of a triggered phosphate sensor $7,50,51$. Second, a hydrophobic environment induces the external presentation of hydrophobic residues of the finger loop on the central crest of the arrestin molecule, which folds into a short $\alpha$-helix ${ }^{13,28}$. Our data suggest that this helical finger loop is a hallmark of the triggered activation sensor. Finally, both receptor-dependent and receptor-independent arrestin activation induce an inter-domain twist in $\mathrm{IP}_{6}$-bound arrestin-3 (Fig. 1b) and all other activated arrestin structures ${ }^{10-13}$.

Molecular mechanisms connecting the phosphate and activation sensors to the domain twist have not been proposed. Our data suggest that each sensor acts via a distinct pathway. Triggering the phosphate sensor likely facilitates the inter-domain rotation in two ways. First, $\mathrm{IP}_{6}$ phosphates bind Lys295 on the lariat loop (Fig. 2a). This changes the loop position, removing two negative charges from the polar core (Fig. $2 \mathrm{a}, \mathrm{b}$ ). Second, $\mathrm{IP}_{6}$ directly displaces the C-tail, which contributes Arg393 to the polar core (Fig. 2b). Both changes break the polar core, making domain rotation possible.

Triggering the activation sensor likely promotes inter-domain rotation by a complementary mechanism. An interaction between the finger loop and a hydrophobic environment can induce helix formation. The finger loop is part of the N-domain, but in basal arrestin, the hydrophobic residues are shielded from solvent via an interaction with a loop in the C-domain (residues 243-247, Fig. 6a, b). Activation-associated rearrangement of the finger loop alters this hydrophobic core, so that the $\beta$-strands surrounding the finger loop in the basal state shift in a manner promoting the domain rotation (Fig. 6a).

Based on this model, we propose how arrestins have achieved an inter-domain twist in reported structures (Supplementary Table 2). Our analysis suggests that in the $\mathrm{IP}_{6}$-bound arrestin-3, a physiologically relevant activator triggers both sensors, which appear to be highly interdependent. During receptor-induced activation, the sensors may act more independently, with some GPCRs relying more heavily on one sensor ${ }^{33}$. However, the two sensors likely act synergistically, as arrestins exhibit the highest binding when receptor is both activated and phosphorylated ${ }^{5}$.

The comparison of $\mathrm{IP}_{6}$-activated arrestin-3 and other active arrestin structures suggests a general model explaining how activated arrestins initiate and direct signaling. First, the interdomain twist and the new positions of switches likely work in conjunction to form effector-binding sites. Because receptorindependent activation apparently biases signaling toward the JNK3 cascade ${ }^{15-18,21}, \mathrm{IP}_{6}$-activated arrestin-3 is the first case where the signaling bias can be correlated with the structure. The inspection of other active arrestin structures suggests an intriguing possible mechanism of signal bias. The inter-domain twist is of different magnitude in available active structures, consistent with the proposed ability of active arrestin to adopt a range of conformations $9,52,53$. Moreover, conformations of the switch regions in our structure differ from those observed in other active arrestins. This ability to adopt a range of active conformations agrees with electron microscopy images of a chimeric $\beta_{2}$-adrenergic receptor with arrestin- $2^{54}$, fluorescence quenching binding studies of rhodopsin-arrestin- $1^{55}$ and NMR spectroscopy of rhodopsin-arrestin- $1^{29}$, all of which suggest heterogeneity of the receptor-arrestin complex.

Conceivably, signaling toward different effectors can be directed by combining distinct conformations of the arrestin switch regions with different inter-domain rotation angles (Fig. 6c-e, Supplementary Movies 1 and 2). The magnitude of each conformational change likely depends on the identity of the activator. In case of GPCRs, the conformations may 
be further influenced by the phosphorylation pattern of the receptor $52,53,56,57$. Structures of arrestin in complex with a range of activators, including receptors with different phosphorylation patterns, are required to test this idea.

\section{Methods}

Materials. DNA modifying enzymes were from New England Biolabs (Ipswich, MA). DNA purification kits were from Zymo Research (Irvine, CA). HEK293 and COS7 cells are from ATCC. Cell culture reagents and media were from Mediatech (Manassas, VA) or Invitrogen (Carlsbad, CA). The luciferase substrate coelenterazine- $h$ was from NanoLight (Pinetop, AZ). All other reagents were from Amresco (Solon, OH) or Sigma-Aldrich (St. Louis, MO). The primers used in this study are listed in Supplementary Table 3.

Arrestin-3 expression and purification. Bovine arrestin- 3 was cloned into the pTrcHisB vector (Invitrogen; Carlsbad, CA) with the codon for L394 mutagenized to a stop codon (TGA). This created a version of arrestin- 3 truncated immediately after the C-tail attachment point (encoding residues 1-393 and deleting residues 394-408). This version of arrestin-3 has activation propensity indistinguishable from wild-type and was used to produce protein for the structure of the basal conformation of arrestin $-3^{6}$. Because it has superior properties after purification, arrestin-3 (1-393) was used for the in vitro experiments in the manuscript, with the exception of a subset of the replicates that tested the effects of benzamidine. Only for these benzamidine experiments, one of the replicates arrestin-3 with a stop codon introduced at position R393. This variant of arrestin (1-392) is more easily activated. Experiments in cells used full-length arrestin-3 (1-408).

The pTrcHisB plasmid containing arrestin-3 (1-393) was transformed into E. coli BL21 Gold and grown in $1 \mathrm{~L}$ cultures in LB medium supplemented with $100 \mathrm{mg} / \mathrm{L}$ ampicillin. Cells were grown at $30^{\circ} \mathrm{C}$ overnight with shaking at 250 r.p. $\mathrm{m}$., then protein expression was induced with the addition of $35 \mu \mathrm{M}$ IPTG for $4 \mathrm{~h}$. Cells were collected by centrifugation and the pellet was stored at $-80{ }^{\circ} \mathrm{C}$

Arrestin-3 was purified using a modification of a previously described protocol $^{58}$. Briefly, cell pellets were resuspended in buffer containing $50 \mathrm{mM}$ MOPS, pH 7.2, 5 mM EGTA, 2 mM tris-(2-carboxyethyl) phosphine (TCEP), and two protease inhibitor cocktail tablets (Sigma). Cells were disrupted by sonication at $4{ }^{\circ} \mathrm{C}$. The lysate was clarified by centrifugation at $20,800 \times g$ (SLA 3000 rotor, RC5B Plus centrifuge) for $60 \mathrm{~min}$, and arrestin was precipitated by the addition of $\left(\mathrm{NH}_{4}\right)_{2} \mathrm{SO}_{4}$ to a final concentration $0.32 \mathrm{mg} / \mathrm{mL}$. Precipitated arrestin-3 was collected by centrifugation at $20,800 \times g$ (SLA 3000 rotor, RC-5B Plus centrifuge) for $90 \mathrm{~min}$, and dissolved in buffer containing $10 \mathrm{mM}$ MOPS pH 7.2, $2 \mathrm{mM}$ EGTA, and $1 \mathrm{mM}$ TCEP, then centrifuged again at 20,800 $\times$ (SLA 3000 rotor, RC-5B Plus centrifuge) for $60 \mathrm{~min}$ to remove particulates. The supernatant containing soluble arrestin-3 was applied onto a heparin column and eluted with a linear $\mathrm{NaCl}$ gradient. Fractions containing arrestin- 3 were identified by SDS-PAGE and Western and combined. The salt concentration of the pooled fractions was adjusted to $100 \mathrm{mM}$, and the solution was loaded onto a linked HiTrap Q HP (GE healthcare) and HiTrap SP HP (GE healthcare) column. At a NaCl concentration of $100 \mathrm{mM}$, arrestin-3 flows through the $\mathrm{Q}$ column (while most contaminants bind), but binds the SP column. The columns were uncoupled and a linear $\mathrm{NaCl}$ gradient was used to elute arrestin-3 from the SP column. The fractions containing arrestin-3 were identified by SDS-PAGE and combined, concentrated with a $30 \mathrm{kDa}$ cutoff concentrator, then further purified using a Superdex 200 increase 10/ 300 GL column (GE healthcare) equilibrated with $20 \mathrm{mM}$ MOPS pH 7.2, $150 \mathrm{mM}$ $\mathrm{NaCl}$, and $2 \mathrm{mM}$ TCEP. Folding and trimerization were monitored by size exclusion chromatography.

Crystallography. Purified arrestin-3 (residues 1-393) was concentrated to $5 \mathrm{mg} /$ $\mathrm{mL}$ and incubated with $\mathrm{IP}_{6}$ at 1:20 molar ratio for $30 \mathrm{~min}$ on ice. Crystals in the hexagonal space group $\mathrm{P}_{3}$ were grown using the sitting drop vapor diffusion method by combining $2 \mu \mathrm{l}$ of arrestin-3-IP $\mathrm{IP}_{6}$ and $2 \mu \mathrm{l}$ of reservoir solution $(100 \mathrm{mM}$ Succinate/Phosphate/Glycine pH 8.5 and 25\% PEG 1500). Microcrystals appeared within $24 \mathrm{~h}$ and were used for seeding. Crystals were harvested after 7 days, cryoprotected in $50 \% \mathrm{w} / \mathrm{v}$ glycerol and cryocooled by plunging into liquid nitrogen.

Diffraction data (Table 1) were collected at the Advanced Photon Source LSCAT beamline 21-ID-D. Data were processed using HKL $2000^{59}$. The structure was determined by molecular replacement in PHASER ${ }^{60}$ using isolated domains of a pruned version of arrestin-2 (PDB entry $1 \mathrm{G}_{4} \mathrm{M}^{4}$ ) as the search model. The best solution was associated with an initial $\mathrm{R} / \mathrm{R}_{\text {free }}$ of $0.34 / 0.37$ and was improved by model building in COOT $^{61}$ and refinement in PHENIX ${ }^{62}$. The myoD conformation of $\mathrm{IP}_{6}$ was placed into difference electron density manually using the conformation found in PDB entry 4HNW. Figures were prepared in PYMOL.

JNK3 activation measurements in HEK293 and COS7 cells. HA-tagged ASK1 and Flag-tagged JNK3 were co-transfected with either: (1) empty vector; (2) wildtype arrestin-3 (residues 1-408); or the indicated arrestin-3 mutants (residues 1-408). Lipofectamine2000 (Thermo Fisher) was used for transfection. After 48hours, cells were incubated with phosphatase inhibitors $(50 \mathrm{mM} \mathrm{NaF}$ and $10 \mathrm{mM}$ $\mathrm{Na}_{3} \mathrm{VO}_{4}$ ) in PBS for $15 \mathrm{~min}$ at $37^{\circ} \mathrm{C}$ and lysed with lysis buffer containing $50 \mathrm{mM}$
Tris pH7.8, 2 mM EDTA, $250 \mathrm{mM} \mathrm{NaCl}, 10 \%$ glycerol, 0.5\% NP-50, $20 \mathrm{mM} \mathrm{NaF}$, $1 \mathrm{mM} \mathrm{Na}_{3} \mathrm{VO}_{4}, 1 \mathrm{mM}$ phenylmethanesulfonyl fluoride (PMSF) and $2 \mathrm{mM}$ benzamidine. Sonication was used to further lyse the cells (60 Sonic Dismembrator, Fisher Scientific). The whole cell lysate were centrifuged at $10,000 \times \mathrm{g}$ for $15 \mathrm{~min}$ and the supernatant was used for Western analysis. Activation of JNK3 was assessed using a phospho-JNK specific antibody (Cell Signaling \#9251, 1:1000 dilution). The expression level of HA-ASK1, Flag-JNK3 and arrestin-3 were assessed by Western analysis using antibodies against the HA tag (Cell Signaling \#C29F4, 1:1000 dilution), the Flag tag (Sigma \#F3165, 1:500 dilution), arrestin (F4C1) ${ }^{63}$ or GFP (JL-8, Choltech \#632381, 1:2000 dilution), respectively. The results were quantified using VersaDoc and QuantityOne software (Bio-Rad). The uncropped blots of one representative experiment are shown in Supplementary Fig. 8.

DEER distance measurements. Cysteine substitutions of Ser13 and Ala392 were introduced into otherwise Cys-less arrestin-3 (residues 1-393). This variant was purified and spin-labeled with MTSL, as described previously ${ }^{31}$. Double electron electron resonance (DEER) spectroscopy data were collected using a Bruker E580 operating at Q-band and equipped with an EN5107D2 resonator. Samples contained $20 \%$ deuterated glycerol as a cryoprotectant and $100 \mu \mathrm{M}$ protein with varying amounts of $\mathrm{IP}_{6}$ were run at $80 \mathrm{~K}$ following flash freezing in a dry ice and acetone mixture. Acquired raw dipolar evolution data were phase and background corrected, plotted and analyzed for distance distributions in the same way for each data set using the algorithms included in the LongDistances software program ${ }^{64}$ written by C. Altenbach (University of California-Los Angeles, CA). The upper reliable distance limit of $65 \AA$ was determined based on the maximum data collection time $(t=4.5 \mu \mathrm{s})$ of the DEER experiments according to the equation $d \approx 5$ $(t / 2) 1 / 3^{65}$ and is reflected in the $x$-axis of the distance distribution plot. The release of the C-tail causes the distances to spread out and possibly become longer than the observable range of our data.

Microscale thermophoresis. Microscale thermophoresis (MST) was conducted using a NT.115 MST instrument (NanoTemper Technologies GmbH) equipped with green and blue filter sets. All arrestin-3 variants (residues 1-393; $20 \mu \mathrm{M}$ in MST buffer (20 mM 3-morpholinopropane-1-sulfonic acid (MOPS) pH 7.5, $150 \mathrm{mM} \mathrm{NaCl}$, and $1 \mathrm{mM} \mathrm{TCEP}$ ) were labeled by adding $200 \mu \mathrm{L}$ of $20 \mu \mathrm{M}$ arrestin3 to $1 \mu \mathrm{L}$ of $40 \mathrm{mM}$ Alexa Fluor C5 maleimide dye (final dye concentration, 200 $\mu \mathrm{M}$; Molecular Probes, Eugene, OR) in DMSO and incubating the mixture at room temperature in the dark for $30 \mathrm{~min}$. Free dye was separated from the protein-dye conjugate using a PD10 G25 column (GE Healthcare Bio-Sciences, Piscataway, NJ) equilibrated in MST buffer. Lauryl maltose neopentyl glycol (MNG-3; Anatrace, Maumee, $\mathrm{OH})$ at $1 \%(\mathrm{w} / \mathrm{v})$ in all solutions prevented the adherence of protein to the plasticware. The labeling stoichiometry was $0.95-2.0$ dye molecules per arrestin-3 as estimated by spectrophotometry.

Titrations were usually accomplished by preparing 15 samples of $\mathrm{IP}_{6}$ in a 1:1 dilution scheme. A sixteenth sample with no $\mathrm{IP}_{6}$ established the baseline fluorescence or temperature jump. Labeled arrestin-3 $(50 \mathrm{nM})$ and the reaction mixtures were equilibrated for 45 min then loaded into premium coated capillary tubes (NanoTemper). Data were acquired using 40\% MST and 50\% LED settings. A $5 \mathrm{~s}$ pre-IR phase was recorded, followed by a $60 \mathrm{sec}$ phase with the IR laser on and a $5 \mathrm{~s}$ post-IR phase. In the case of the wild-type arrestin- 3 construct, the pre-IR fluorescence varied strongly ( $\sim 30 \%$ difference between the maximum and minimum intensity values) as a function of $\mathrm{IP}_{6}$ concentration, allowing this signal to be analyzed directly. The temperature-jump methodology was used to analyze data from the $\Delta \mathrm{N}_{\mathrm{IPG}}$ or T222C variants ${ }^{66}$. To prevent aggregation of the latter mutant, we included $0.5 \mathrm{mg} / \mathrm{mL}$ soybean trypsin inhibitor (Worthington Biochemical) in the MST buffer; this mutant also required an adjustment of the illumination protocol, featuring 75\% LED power and an IR-on phase of only $30 \mathrm{~s}$ Data were the average of at least two replicates. Data were analyzed in a version of PALMIST $^{66}$ modified to include two-site binding models (manuscript submitted). MST figures were rendered using GUSSI ${ }^{67}$.

Analytical ultracentrifugation. Purified arrestin-3 (residues 1-393) with or without $\mathrm{IP}_{6}(100 \mu \mathrm{M})$ was analyzed in an Optima XLI ultracentrifuge (Beckman Coulter, Brea, CA) equipped with a four-hole An- $60 \mathrm{Ti}$ rotor at $142,000 \times g$ at $4{ }^{\circ} \mathrm{C}$ Samples were loaded into double-sector cells (path length of $1.2 \mathrm{~cm}$ ) with charcoalfilled Epon centerpieces and sapphire windows. Sedfit (version 12.0) was used to analyze velocity scans using every scan from a total of between $250-300$ scans $^{68}$. Approximate size distributions were determined for a confidence level of $p=0.95$, a resolution of $n=300$, and sedimentation coefficients between 0.1 and $15 \mathrm{~S}$. The frictional ratio was allowed to float.

BRET measurements of arrestin-3 binding to receptors. Interactions between N-terminally Venus-tagged arrestin-3 (residues 1-408) and C-terminally RLuc8tagged M2 muscarinic receptor or D2 dopamine receptor were determined by BRET, as described, using the highest (saturating) arrestin- 3 concentrations ${ }^{14}$ Absolute levels of luminescence were used as the measure of the expression levels of RLuc-tagged receptors, whereas direct fluorescence was used to determine the expression of Venus-tagged arrestins, as described ${ }^{14}$. BRET was measured $15 \mathrm{~min}$ after the addition of $10 \mu \mathrm{M}$ M2 agonist carbachol or $10 \mu \mathrm{M}$ D2 agonist quinpirole, 
both of which were added along with $5 \mu \mathrm{M}$ of the luciferase substrate coelenterazine-h. Luminescence and fluorescence were measured using Infinite F500 multimode plate reader (Tecan). Net BRET was calculated as the difference between BRET signal in the presence and absence of agonist to determine the expression of Venus-tagged arrestins, as described ${ }^{14}$. BRET was measured $15 \mathrm{~min}$ after the addition of $10 \mu \mathrm{M}$ M2 agonist carbachol or $10 \mu \mathrm{M}$ D2 agonist quinpirole, both of which were added in conjunction with $5 \mu \mathrm{M}$ of the luciferase substrate coelenterazine-h. Luminescence and fluorescence were measured using Infinite F500 multimode plate reader (Tecan). Net BRET was calculated as the difference between BRET signal in the presence and absence of agonist.

JNK3 binding of arrestin-3 variants. His tagged JNK3 $22(10 \mu \mathrm{g})$ was incubated with Ni-NTA resin for $2 \mathrm{~h}$ and wild-type or disulfide-containing arrestin-3 $(10 \mu \mathrm{g})$ was added to the mixture with and without $100 \mu \mathrm{M} \mathrm{IP}_{6}$. The samples were washed with buffer (20 mM MOPS, pH 7.4, $150 \mathrm{mM} \mathrm{NaCl}, 25 \mathrm{mM}$ imidazole) and eluted with $100 \mu \mathrm{l}$ of the same buffer containing $250 \mathrm{mM}$ imidazole. The eluate was methanol precipitated, and the JNK3 bound arrestin-3 (residues 1-393) was visualized by both Coomassie blue staining and Western blot, quantified using the QuantityOne software, and the data were analyzed using Prism. For the disulfidelinked bands, measurements were made in quadruplicate and one-way ANOVA was used to compare JNK3 binding to disulfide-containing arrestin-3. The uncropped blots of one representative experiment are shown in Supplementary Fig. 8.

Data availability. The authors declare that all data supporting the findings of this study are available within the article and its Supplementary Information files. Coordinates and structure factors have been deposited in the Protein Data Bank with accession code 5TV1. Raw diffraction data are deposited with SBGrid with the accession code 10.15785/SBGRID/330. The additional data that support the findings of this study are available from the corresponding authors upon request.

Received: 4 January 2017 Accepted: 29 August 2017

Published online: 10 November 2017

\section{References}

1. Wilden, U., Hall, S. W. \& Kuhn, H. Phosphodiesterase activation by photoexcited rhodopsin is quenched when rhodopsin is phosphorylated and binds the intrinsic $48-\mathrm{kD}$ a protein of rod outer segments. Proc. Natl Acad. Sci. USA 83, 1174-1178 (1986).

2. DeWire, S. M., Ahn, S., Lefkowitz, R. J. \& Shenoy, S. K. Beta-arrestins and cell signaling. Annu. Rev. Physiol. 69, 483-510 (2007).

3. Xiao, K. et al. Functional specialization of beta-arrestin interactions revealed by proteomic analysis. Proc. Natl Acad. Sci. USA 104, 12011-12016 (2007).

4. Han, M., Gurevich, V. V., Vishnivetskiy, S. A., Sigler, P. B. \& Schubert, C. Crystal structure of beta-arrestin at 1.9 A: possible mechanism of receptor binding and membrane translocation. Strucutre 9, 869-880 (2001).

5. Gurevich, V. V. \& Benovic, J. L. Visual arrestin interaction with rhodopsin. Sequential multisite binding ensures strict selectivity toward light-activated phosphorylated rhodopsin. J. Biol. Chem. 268, 11628-11638 (1993).

6. Zhan, X., Gimenez, L. E., Gurevich, V. V. \& Spiller, B. W. Crystal structure of arrestin-3 reveals the basis of the difference in receptor binding between two non-visual subtypes. J. Mol. Biol. 406, 467-478 (2011).

7. Hirsch, J. A., Schubert, C., Gurevich, V. V. \& Sigler, P. B. The 2.8 A crystal structure of visual arrestin: a model for arrestin's regulation. Cell 97, 257-269 (1999).

8. Sutton, R. B. et al. Crystal structure of cone arrestin at $2.3 \mathrm{~A}$ : evolution of receptor specificity. J. Mol. Biol. 354, 1069-1080 (2005).

9. Gurevich, V. V. \& Gurevich, E. V. The structural basis of arrestin-mediated regulation of G-protein-coupled receptors. Pharmacol. Ther. 110, 465-502 (2006).

10. Kim, Y. J. et al. Crystal structure of pre-activated arrestin p44. Nature 497, 142-146 (2013)

11. Shukla, A. K. et al. Structure of active beta-arrestin-1 bound to a G-proteincoupled receptor phosphopeptide. Nature 497, 137-141 (2013).

12. Granzin, J., Stadler, A., Cousin, A., Schlesinger, R. \& Batra-Safferling, R. Structural evidence for the role of polar core residue Arg175 in arrestin activation. Sci. Rep. 5, 15808 (2015).

13. Kang, Y. et al. Crystal structure of rhodopsin bound to arrestin determined by femtosecond X-ray laser. Nature 523, 561-567 (2015).

14. Breitman, M. et al. Silent scaffolds: inhibition OF c-Jun N-terminal kinase 3 activity in cell by dominant-negative arrestin-3 mutant. J. Biol. Chem. 287, 19653-19664 (2012)

15. McDonald, P. H. et al. Beta-arrestin 2: a receptor-regulated MAPK scaffold for the activation of JNK3. Science 290, 1574-1577 (2000).
16. Miller, W. E. \& Lefkowitz, R. J. Expanding roles for beta-arrestins as scaffolds and adapters in GPCR signaling and trafficking. Curr. Opin. Cell. Biol. 13, 139-145 (2001).

17. Zhan, X., Kaoud, T. S., Kook, S., Dalby, K. N. \& Gurevich, V. V. JNK3 enzyme binding to arrestin- 3 differentially affects the recruitment of upstream mitogenactivated protein (MAP) kinase kinases. J. Biol. Chem. 288, 28535-28547 (2013).

18. Zhan, X., Kook, S., Gurevich, E. V. \& Gurevich, V. V. Arrestin-dependent activation of JNK family kinases. Handb. Exp. Pharmacol. 219, 259-280 (2014).

19. Palczewski, K., Pulvermuller, A., Buczylko, J. \& Hofmann, K. P. Phosphorylated rhodopsin and heparin induce similar conformational changes in arrestin. $J$. Biol. Chem. 266, 18649-18654 (1991).

20. Zhan, X., Perez, A., Gimenez, L. E., Vishnivetskiy, S. A. \& Gurevich, V. V. Arrestin-3 binds the MAP kinase JNK3alpha2 via multiple sites on both domains. Cell. Signal. 26, 766-776 (2014)

21. Song, X., Coffa, S., Fu, H. \& Gurevich, V. V. How does arrestin assemble MAPKs into a signaling complex? J. Biol. Chem. 284, 685-695 (2009).

22. Bunce, C. M. et al. Comparison of the levels of inositol metabolites in transformed haemopoietic cells and their normal counterparts. Biochem. J. 289, 667-673 (1993). (Pt 3).

23. Sasakawa, N., Sharif, M. \& Hanley, M. R. Metabolism and biological activities of inositol pentakisphosphate and inositol hexakisphosphate. Biochem. Pharmacol. 50, 137-146 (1995).

24. Milano, S. K., Kim, Y. M., Stefano, F. P., Benovic, J. L. \& Brenner, C. Nonvisual arrestin oligomerization and cellular localization are regulated by inositol hexakisphosphate binding. J. Biol. Chem. 281, 9812-9823 (2006).

25. Farrens, D. L., Altenbach, C., Yang, K., Hubbell, W. L. \& Khorana, H. G. Requirement of rigid-body motion of transmembrane helices for light activation of rhodopsin. Science 274, 768-770 (1996).

26. Scheerer, P. et al. Crystal structure of opsin in its G-protein-interacting conformation. Nature 455, 497-502 (2008).

27. Rasmussen, S. G. et al. Crystal structure of the beta2 adrenergic receptor-Gs protein complex. Nature 477, 549-555 (2011).

28. Szczepek, M. et al. Crystal structure of a common GPCR-binding interface for G protein and arrestin. Nat. Commun. 5, 4801 (2014).

29. Zhuang, T. et al. Involvement of distinct arrestin-1 elements in binding to different functional forms of rhodopsin. Proc. Natl Acad. Sci. USA 110, 942-947 (2013).

30. Krissinel, E. B. \& Henrick, K. Inference of macromolecular assemblies from crystalline state. J. Mol. Biol. 372, 774-797s (2007).

31. Zhuo, Y., Vishnivetskiy, S. A., Zhan, X., Gurevich, V. V. \& Klug, C. S. Identification of receptor binding-induced conformational changes in nonvisual arrestins. J. Biol. Chem. 289, 20991-21002 (2014).

32. Chen, Q. et al. Self-association of arrestin family members. Handb. Exp. Pharmacol. 219, 205-223 (2014).

33. Gimenez, L. E. et al. Role of receptor-attached phosphates in binding of visual and non-visual arrestins to G protein-coupled receptors. J. Biol. Chem. 287, 9028-9040 (2012).

34. Gimenez, L. E., Babilon, S., Wanka, L., Beck-Sickinger, A. G. \& Gurevich, V. V. Mutations in arrestin-3 differentially affect binding to neuropeptide $\mathrm{Y}$ receptor subtypes. Cell Signal. 26, 1523-1531 (2014).

35. Milano, S. K., Pace, H. C., Kim, Y. M., Brenner, C. \& Benovic, J. L. Scaffolding functions of arrestin-2 revealed by crystal structure and mutagenesis. Biochemistry 41, 3321-3328 (2002).

36. Luttrell, L. M. et al. Beta-arrestin-dependent formation of beta2 adrenergic receptor-Src protein kinase complexes. Science 283, 655-661 (1999).

37. Vey, J. L. et al. Structure and mechanism of ORF36, an amino sugar oxidizing enzyme in everninomicin biosynthesis. Biochemistry 49, 9306-9317 (2010).

38. Bruender, N. A., Thoden, J. B. \& Holden, H. M. X-ray structure of kijd3, a key enzyme involved in the biosynthesis of D-kijanose. Biochemistry 49, 3517-3524 (2010).

39. Vishnivetskiy, S. A., Hirsch, J. A., Velez, M. G., Gurevich, Y. V. \& Gurevich, V. $\mathrm{V}$. Transition of arrestin into the active receptor-binding state requires an extended interdomain hinge. J. Biol. Chem. 277, 43961-43967 (2002).

40. Hanson, S. M. et al. Arrestin mobilizes signaling proteins to the cytoskeleton and redirects their activity. J. Mol. Biol. 368, 375-387 (2007).

41. Johnson, E., Nguyen, P. T., Yeates, T. O. \& Rees, D. C. Inward facing conformations of the MetNI methionine ABC transporter: implications for the mechanism of transinhibition. Protein Sci. 21, 84-96 (2012).

42. Renault, L., Guibert, B. \& Cherfils, J. Structural snapshots of the mechanism and inhibition of a guanine nucleotide exchange factor. Nature 426, 525-530 (2003).

43. Westblade, L. F. et al. Structural basis for the bacterial transcription-repair coupling factor/RNA polymerase interaction. Nucleic Acids Res. 38, 8357-8369 (2010). 
44. Shoemaker, B. A., Portman, J. J. \& Wolynes, P. G. Speeding molecular recognition by using the folding funnel: the fly-casting mechanism. Proc. Natl Acad. Sci. USA 97, 8868-8873 (2000).

45. Sugase, K., Dyson, H. J. \& Wright, P. E. Mechanism of coupled folding and binding of an intrinsically disordered protein. Nature 447, 1021-1025 (2007).

46. Hanson, S. M. et al. Differential interaction of spin-labeled arrestin with inactive and active phosphorhodopsin. Proc. Natl Acad. Sci. USA 103, 4900-4905 (2006).

47. Vishnivetskiy, S. A. et al. The role of arrestin alpha-helix I in receptor binding. J. Mol. Biol. 395, 42-54 (2010).

48. Laporte, S. A. et al. The beta2-adrenergic receptor/betaarrestin complex recruits the clathrin adaptor AP-2 during endocytosis. Proc. Natl Acad. Sci. USA 96, 3712-3717 (1999).

49. Kang, D. S. et al. Structure of an arrestin-2-clathrin complex reveals a novel clathrin binding domain that modulates receptor trafficking. J. Biol. Chem. 284, 29860-29872 (2009).

50. Vishnivetskiy, S. A. et al. How does arrestin respond to the phosphorylated state of rhodopsin? J. Biol. Chem. 274, 11451-11454 (1999).

51. Vishnivetskiy, S. A. et al. An additional phosphate-binding element in arrestin molecule. Implications for the mechanism of arrestin activation. J. Biol. Chem. 275, 41049-41057 (2000).

52. Kim, J. et al. Functional antagonism of different G protein-coupled receptor kinases for beta-arrestin-mediated angiotensin II receptor signaling. Proc. Natl Acad. Sci. USA 102, 1442-1447 (2005).

53. Ren, X. R. et al. Different G protein-coupled receptor kinases govern G protein and beta-arrestin-mediated signaling of V2 vasopressin receptor. Proc. Natl Acad. Sci. USA 102, 1448-1453 (2005).

54. Shukla, A. K. et al. Visualization of arrestin recruitment by a G-protein-coupled receptor. Nature 512, 218-222 (2014).

55. Sinha, A., Jones Brunette, A. M., Fay, J. F., Schafer, C. T. \& Farrens, D. L. Rhodopsin TM6 can interact with two separate and distinct sites on arrestin: evidence for structural plasticity and multiple docking modes in arrestinrhodopsin binding. Biochemistry 53, 3294-3307 (2014).

56. Yang, F. et al. Phospho-selective mechanisms of arrestin conformations and functions revealed by unnatural amino acid incorporation and (19)F-NMR. Nat. Commun. 6, 8202 (2015).

57. Nobles, K. N. et al. Distinct phosphorylation sites on the beta(2)-adrenergic receptor establish a barcode that encodes differential functions of beta-arrestin. Sci. Signal. 4, ra51 (2011).

58. Vishnivetskiy, S. A., Zhan, X., Chen, Q., Iverson, T. M. \& Gurevich, V. V. Arrestin expression in E. coli and purification. Curr. Protoc. Pharmacol. 67, Unit 2 11(2014)

59. Otwinowski, Z. \& Minor, W. Processing of X-ray diffraction data collected in oscillation mode. Methods Enzymol. 276, 307-326 (1997).

60. McCoy, A. J. et al. Phaser crystallographic software. J. Appl. Crystallogr. 40, 658-674 (2007).

61. Emsley, P. \& Cowtan, K. Coot: model-building tools for molecular graphics. Acta Crystallogr. D Biol. Crystallogr. 60, 2126-2132 (2004).

62. Adams, P. D. et al. PHENIX: a comprehensive Python-based system for macromolecular structure solution. Acta. Crystallogr. D Biol. Crystallogr. 66, 213-221 (2010)

63. Donoso, L. A. et al. S-antigen: preparation and characterization of site-specific monoclonal antibodies. Curr. Eye Res. 9, 343-355 (1990).

64. Toledo Warshaviak, D., Khramtsov, V. V., Cascio, D., Altenbach, C. \& Hubbell, W. L. Structure and dynamics of an imidazoline nitroxide side chain with strongly hindered internal motion in proteins. J. Magn. Reson. 232, 53-61 (2013).

65. Jeschke, G. DEER distance measurements on proteins. Annu. Rev. Phys. Chem. 63, 419-446 (2012).

66. Scheuermann, T. H., Padrick, S. B., Gardner, K. H. \& Brautigam, C. A. On the acquisition and analysis of microscale thermophoresis data. Anal. Biochem. 496, 79-93 (2016).

67. Brautigam, C. A. Calculations and publication-quality illustrations for analytical ultracentrifugation data. Methods Enzymol. 562, 109-133 (2015).
68. Schuck, P. Size-distribution analysis of macromolecules by sedimentation velocity ultracentrifugation and lamm equation modeling. Biophys. J. 78, 1606-1619 (2000).

\section{Acknowledgements}

We thank Drs B. Spiller, J. York, H. Hamm, C. Sanders, W. Hubbell, C. Goodman, and K. McCulloch for critical reading and insightful discussions, H.E. Xu for an advance on the rhodopsin-arrestin-1 coordinates, S. Prokop, I. Yamakawa, M. Schoenberger, K. McCulloch, X. Zhan. M. Tyska, H. McDonald, and K. Schultz for experimental assistance. This work was supported by NIH grants to VVG (GM077561, GM109955 (these two R01 grants were replaced by R35 GM122491), EY011500) and TMI (GM095633, GM120569), and TMI/VVG (DA043680), a Vanderbilt Discovery Grant (TMI/VVG), and a Vanderbilt Vision Research Center NEI Core Grant (EY008126). Q.C. was supported by the Vanderbilt International Scholars Program. N.A.P. was supported by T32 GM007628 and the American Heart Association (16PRE30180007). NCG was supported by the American Heart Association (13POST16910057). J.T. was supported by a DAAD RISE fellowship. The Vanderbilt crystallization facility is supported by S10 RR026915. A.U.C. is supported by the Vanderbilt Center for Structural Biology. The Advanced Photon Source, a User Facility operated for the U.S. DOE Office of Science, was supported under Contract DE-AC02-06CH11357. LS-CAT Sector 21 is supported by the Michigan Economic Development Corporation and the Michigan Technology TriCorridor (085P1000817). DEER instrumentation is supported by S10RR022422 and S10OD011937, use of the National Biomedical EPR Center is supported by P41EB001980.

\section{Author contributions}

Q.C. performed protein purification, crystallography, SEC measurements, and $\mathrm{IP}_{6}$ dependent JNK3 activation. N.C.G. assisted with crystallography. N.A.P. and J.T. performed BRET assays. S.B. performed JNK3 binding assays, E.V.G. performed JNK3 activation assays. Y.Z. and C.S.K. performed DEER experiments. S.A.V. and Q.C. performed rhodopsin binding. C.A.B. performed MST analysis. P.K.S. and M.D.O. performed AUC. V.V.G. and T.M.I. developed the study, performed molecular biology and protein purification, and guided the experiments and analysis. Q.C., V.V.G., and T. M.I. wrote the paper with input from the other authors.

\section{Additional information}

Supplementary Information accompanies this paper at doi:10.1038/s41467-017-01218-8.

Competing interests: The authors declare no competing financial interests.

Reprints and permission information is available online at http://npg.nature.com/ reprintsandpermissions/

Publisher's note: Springer Nature remains neutral with regard to jurisdictional claims in published maps and institutional affiliations.

\footnotetext{
Open Access This article is licensed under a Creative Commons Attribution 4.0 International License, which permits use, sharing adaptation, distribution and reproduction in any medium or format, as long as you give appropriate credit to the original author(s) and the source, provide a link to the Creative Commons license, and indicate if changes were made. The images or other third party material in this article are included in the article's Creative Commons license, unless indicated otherwise in a credit line to the material. If material is not included in the article's Creative Commons license and your intended use is not permitted by statutory regulation or exceeds the permitted use, you will need to obtain permission directly from the copyright holder. To view a copy of this license, visit http://creativecommons.org/ licenses/by/4.0/.
}

(c) The Author(s) 2017 